Revue internationale P.M.E.

Économie et gestion de la petite et moyenne entreprise

\title{
Vers un modèle d'émergence de la petite entreprise
}

\section{Alain Capiez et Émile-Michel Hernandez}

Volume 11, numéro 4, 1998

URI : https://id.erudit.org/iderudit/1009050ar

DOI : https://doi.org/10.7202/1009050ar

Aller au sommaire du numéro

Éditeur(s)

Presses de l’Université du Québec

ISSN

0776-5436 (imprimé)

1918-9699 (numérique)

Découvrir la revue

Citer cet article

Capiez, A. \& Hernandez, É.-M. (1998). Vers un modèle d'émergence de la petite entreprise. Revue internationale P.M.E., 11(4), 11-43.

https://doi.org/10.7202/1009050ar

\section{Résumé de l'article}

Si les espoirs mis dans la création d'entreprise n'ont pas toujours été à la hauteur des espérances économiques, la recherche sur les facteurs de succès ou d'échec de la création et sur le devenir de la nouvelle entité reste active. Les auteurs proposent un modèle d'émergence de la petite firme, en utilisant une double approche contingente et processuelle. À partir d'un échantillon de 118 créations récentes, ils mettent en évidence des facteurs explicatifs de l'émergence ainsi que divers comportements stratégiques correspondant aux étapes du processus d'émergence. Il devient ainsi possible de repérer les entreprises qui ont le plus de chances de se développer et de devenir des PME pérennes. 


\title{
Vers un modèle d'émergence de la petite entreprise
}

\author{
Alain CAPIEZ \\ Université d'Angers \\ Émile-Michel HERNANDEZ \\ Université de Nantes ${ }^{1}$
}

\section{MOTS CLÉS}

Comportement de la firme - Contingence - Création d'entreprise

Émergence de la petite entreprise - Entrepreneuriat - Pépinières

Pérennité - Performance - Processus

\begin{abstract}
RÉSUMÉ
Si les espoirs mis dans la création d'entreprise n'ont pas toujours été à la hauteur des espérances économiques, la recherche sur les facteurs de succès ou d'échec de la création et sur le devenir de la nouvelle entité reste active. Les auteurs proposent un modèle d'émergence de la petite firme, en utilisant une double approche contingente et processuelle. À partir d'un échantillon de 118 créations récentes, ils mettent en évidence des facteurs explicatifs de l'émergence ainsi que divers comportements stratégiques correspondant aux étapes du processus d'émergence. II devient ainsi possible de repérer les entreprises qui ont le plus de chances de se développer et de devenir des PME pérennes.
\end{abstract}

\section{ABSTRACT}

The research about the determinants of new firm success is still vivid. The authors present a model of new venture development with both contingent and processual method. From a 118 ventures sample, they point out several motives of rising and some kind of behavior characterizing the small firm development process. Such a model may discriminate potentially successful start-up firms with long-lasting performance.

1. Les auteurs tiennent à remercier les lecteurs anonymes pour la pertinence de leurs remarques et de leurs suggestions. 


\section{RESUMEN}

Los estudios sobre las causas de éxito de las nuevas empresas estan siempre numerosos. Los autores presentan uno modelo de emergencia para las pequeñas empresas, fundado sobre uno metodo causal y procesual. A partir de una muestra de 118 creaciones recientes, ponan en evidencia ciertas causas de emergencia y tambièn varios compartamientos estrategicos; estos compartamientos caracterizan las etapas del proceso de emergencia. Este modelo permite seleccionar empresas que puedan desarrollarse con resultados duraderos.

Une enquête de l'INSEE (Bonnaud, 1994) fait ressortir que sur 229000 créations de très petites entreprises (TPE) en 1987, cinq ans après (1992), on dénombrait 110000 TPE (moins de 10 salariés) et 5000 PME dont 1500 avaient un effectif compris entre 20 et 49 salariés et 500 un effectif supérieur à 50 salariés. Si presque la moitié de ces entreprises se révèle pérenne, mais sans croissance notable, un peu plus de $2 \%$ ont « réussi » et, parmi celles-ci, $10 \%$ peuvent être considérées comme des moyennes entreprises. L'émergence des PME constitue un processus complexe dont nous chercherons à définir les caractéristiques.

Dans la littérature, l'émergence a surtout été abordée sous un angle organisationnel. Ainsi, l' «émergence organisationnelle » est «le processus d'organisation dont le résultat est une nouvelle organisation » (Gartner, 1993). Mais l'émergence peut aussi être appréhendée sous un aspect stratégique ; l' « émergence économique » a été définie comme «l'obtention d'une masse critique nécessaire pour assurer la survie et la pérennité de la TPE en affirmant sa compétitivité dans le ou les domaines d'activités stratégiques (DAS) visés » (Capiez, 1988). En fait, il est difficile de dissocier l'aspect stratégique de l'aspect organisationnel en raison de leur interrelation ; aussi utiliserons-nous le simple terme «émergence », pris essentiellement dans un sens stratégique, mais sans négliger les aspects organisationnels.

C'est au processus d'émergence que nous nous intéresserons et au rôle du créateur dans ce processus, car c'est le créateur qui détermine le comportement

\section{LES AUTEURS}

Alain Capiez est professeur agrégé en sciences de gestion; il enseigne à l'Université d'Angers et dirige actuellement le DESS « audit et gestion des risques ». Il est l'auteur d'un manuel de gestion financière et de nombreux articles sur la finance d'entreprise et le développement des petites firmes. Adresse: Faculté de droit, d'économie et de sciences sociales, 13, allée François-Mitterrand, B.P. 363, 49036 Angers Cedex 01, France. Téléphone : 02.41.96.21.35. Télécopieur: 02.41.96.21.96. Courriel : alain.capiez@univ-angers.fr

Émile-Michel Hernandez est maître de conférences en sciences de gestion. Après avoir été créateur et dirigeant d'entreprise, il a enseigné aux universités d'Abidjan et de Lomé et est actuellement en poste à l'Université de Nantes. Membre de l'Académie de l'entrepreneuriat, il est l'auteur d'un ouvrage sur le management de l'entreprise africaine et de nombreuses publications sur l'entrepreneuriat. Adresse: IUT de Saint-Nazaire, 58, rue Michel-Ange, B.P. 420, 44606 Saint-Nazaire Cedex. Téléphone: 02.40.17.81.59. Télécopieur: 02.40.17.81.58. 
stratégique de son organisation dont résulte la performance. Aussi, après avoir situé notre recherche dans le contexte entrepreneurial (Saporta, 1994) et posé notre problématique, nous présenterons le modèle utilisé et l'échantillon étudié ; puis nous procéderons à une analyse des résultats unidimensionnelle et multidimensionnelle, et nous terminerons par une discussion et une conclusion sur le processus d'émergence.

\section{Contexte entrepreneurial et problématique de recherche}

L'approche entrepreneuriale de la création d'entreprise souligne l'impulsion donnée par l'entrepreneur à l'organisation. Faite d'abord en termes de contingence, cette approche a évolué vers l'étude du processus.

\subsection{Approche en termes de contingence}

La première approche de la création d'entreprise, dite « fondamentaliste » (Thevenet et Vachette, 1992), considère le créateur comme le fondement de la performance et se focalise sur sa personnalité. Elle trouve son aboutissement dans le modèle de Shapero (1975), mais elle débouche sur une interrogation : faut-il définir le créateur par ce qu'il est (approche par les traits) ou par ce qu'il fait (création d'une organisation) ? Cette controverse est le point de départ d'un courant de recherche organisationnelle sur la création d'entreprise (Carland, Hoy et Carland, 1988 ; Gartner, 1988 ; Gartner, Bird et Starr, 1992). Mais la réalité polymorphe et protéiforme de la création a rendu nécessaire une approche contingente.

La performance de la firme nouvelle (PFN) au regard de la rentabilité ou de la croissance des ventes ou de la part de marché est contingente à des facteurs comme la stratégie (S) et la structure de l'industrie (SI) [Sandberg et Hofer, 1986], ainsi que leur interaction et l'origine de l'entreprise $(\mathrm{O})$ [McDougall, Robinson et DeNisi, 1992]. Aussi le paradigme classique de la recherche entrepreneuriale axée sur l'entrepreneur $(\mathrm{E})$ :

$$
\mathrm{PFN}=\mathrm{f}(\mathrm{E})
$$

a-t-il été remplacé par:

$$
\mathrm{PFN}=\mathrm{f}(\mathrm{E}, \mathrm{O}, \mathrm{S}, \mathrm{SI}, \mathrm{S} \times \mathrm{SI})
$$

La recherche intègre maintenant la dimension stratégique, avec l'identification de facteurs d'environnement hostiles ou favorables au créateur (Hay, Verdin et Williamson, 1993), ou le rapprochement de stratégies types et de choix industriels de localisation afin d'en déduire l'influence sur la performance (McDougall et Robinson, 1990 ; Carter, Stearns, Reynolds et Miller, 1994). 
Si l'approche contingente améliore la compréhension du succès de la création, elle doit être complétée par une approche en termes de processus, car c'est l'évolution de tous ces éléments qui donne son sens à la création.

\subsection{Approche en termes de processus}

Gartner (1988) propose d'aborder le processus de création d'entreprise par une description du travail des entrepreneurs et une analyse systématique des activités, ce qui aboutit à l'étude du comportement organisationnel (organizational behavior), c'est-à-dire des rapports entre le créateur et son organisation. Greenberger et Sexton (1988) expliquent la décision de création par l'interaction d'éléments catalyseurs (vision et personnalité du créateur, degré de contrôle souhaité) et de facteurs externes (vécu d'événements significatifs au plan entrepreneurial, existence d'un support social actif). Guth, Kumaraswany et McErlean (1991) s'attachent au processus cognitif du créateur et Gartner, Bird et Starr (1992), au comportement des acteurs dans les organisations émergentes. Covin et Slevin (1991) appréhendent le processus entrepreneurial sous l'angle du comportement de la firme.

Synthétisant cette approche, Hernandez (1994b) distingue quatre étapes dans le processus de création : l'initiation, la maturation, la décision et la finalisation.

Cette analyse considère les caractéristiques psychosociologiques du créateur comme un fondement de la création, permettant de saisir l'occasion, reconnaît à la stratégie un rôle essentiel dans la réussite future de la firme, met en avant la performance comme condition de pérennité, fait apparaître le caractère diachronique de la création avec des ajustements plus ou moins longs aux circonstances favorables ou aux menaces de l'environnement.

C'est dans l'émergence que le processus entrepreneurial prend tout son sens : « initié par un acte de volonté humaine, il implique un changement d'état, une discontinuité, il est holistique et unique, il implique de nombreuses variables antécédentes, il engendre des résultats extrêmement sensibles aux conditions initiales de ces variables » (Hofer et Bygrave, 1992).

L'émergence correspond essentiellement à la phase de finalisation. L'entreprise en fin de démarrage cherche à affirmer son pouvoir de marché. La durée du démarrage est très variable et peut aller jusqu'à trois ou quatre ans. Kirchloff et Philipps (1989) observent que nombre de jeunes entreprises ne grandissent pas durant leurs quatre premières années d'existence et Woo et al. (1990) montrent que beaucoup de jeunes entreprises changent de gammes de produits, de méthodes de production et de commercialisation pendant les trois premières années. C'est pourquoi nous nous intéresserons à des entreprises dont l'âge est compris entre un et quatre ans et dont on peut penser qu'elles sont en plein processus d'émergence. Comment appréhender l'émergence des petites firmes? 
Schéma 1

Le processus de création d'entreprise

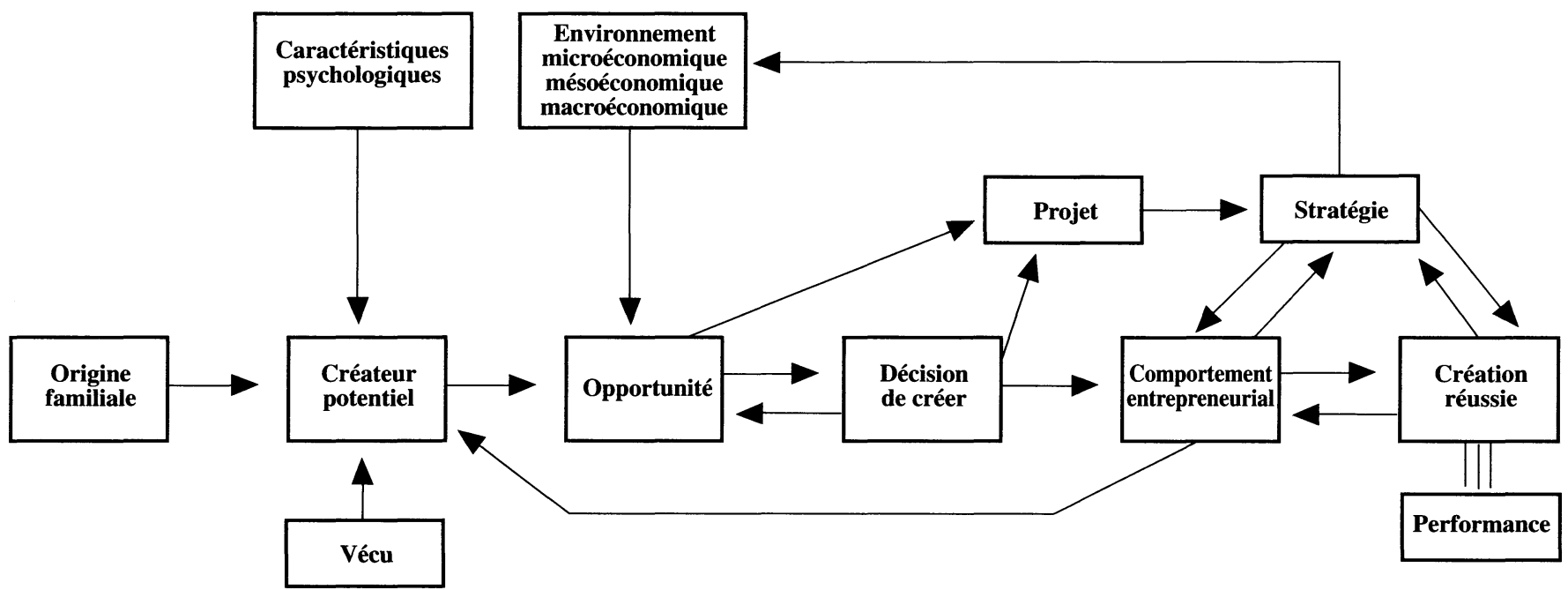

\begin{tabular}{|c|c|c|c|} 
Initiation & Maturation & Décision & Finalisation \\
\hline Étape 1 & Étape 2 & Étape 3
\end{tabular}




\subsection{Problématique de l'émergence}

Notre problématique est la suivante : l'émergence de la petite firme s'explique par la rencontre d'un créateur avec des caractéristiques psychosociologiques déterminant son tempérament et d'une occasion d'affaires, provenant de l'environnement, qu'il a su repérer, voire susciter dans une certaine mesure, ce qui peut être appréhendé par une approche contingente. Mais l'émergence constitue un processus qu'il convient d'analyser. C'est cette analyse qui constitue l'essentiel de notre recherche.

Comment le créateur détermine-t-il le comportement de l'organisation dont il est à l'origine?

Est-il possible de repérer certains comportements types? Certains comportements sont-ils plus aptes que d'autres à assurer la réussite ? À partir de ces comportements, peut-on prévoir l'étendue de la réussite?

Pour apporter des réponses, nous proposons un modèle.

\section{Modèle d'émergence et échantillon}

Nous présenterons les hypothèses et les variables du modèle, puis l'échantillon étudié, avant de préciser la méthode utilisée pour mesurer les variables.

\subsection{Hypothèses et variables du modèle d'émergence}

Le système de la PME peut être présenté comme une interaction entre quatre variables : les buts des dirigeants, l'organisation, les activités et l'environnement (GREPME, 1995). Cette interaction concrétise un comportement de firme que nous avons modélisé en nous inspirant de Covin et Slevin (1991). Nous considérons que :

- Il existe pour la firme comme pour l'individu un comportement entrepreneurial ; « le rôle de l'entrepreneur mis en avant par Schumpeter est socialement vital, mais il peut être rempli par une organisation... Le concept de comportement entrepreneurial de l'entreprise ne constitue pas une rupture radicale avec les aspects plus traditionnels de l'entrepreneuriat, mais une extension logique de la construction de base » (Covin et Slevin, 1991, p. 10). Le comportement entrepreneurial se distingue du comportement organisationnel par la prise de risque, l'innovation et la proactivité. Les firmes à comportement entrepreneurial recherchent plutôt les projets risqués avec une espérance de rentabilité élevée, mettent l'accent sur le leadership technologique, et sont plus dynamiques dans la recherche d'occasions d'affaires.

- Pour la firme en émergence, ce comportement est essentiellement déterminé par le comportement de son créateur. Ce n'est que lorsque l'organisation 
aura pris de l'extension que les comportements peuvent diverger avec l'arrivée d'autres acteurs influents.

- La petite entreprise nouvellement créée doit s'adapter à l'environnement. Mais l'attitude du créateur n'est pas forcément réactive ; elle peut et doit être proactive. Il va ainsi chercher à aménager son environnement par constitution d'un réseau de relations avec les acteurs extérieurs et faire de sa firme un « espace de transaction » très ouvert sur l'environnement (Julien et Marchesnay, 1996).

- La stratégie de la petite entreprise en émergence est essentiellement limitée à la spécialisation, mais la firme dispose de moyens variés pour assurer la spécificité de son offre.

- La petite entreprise recourt peu à la planification formalisée, mais cela ne signifie pas qu'elle n'a pas de stratégie. La planification formalisée au minimum existe : elle constitue un moyen de développer la stratégie du créateur et son formalisme minimum favorise l'adaptabilité.

Le comportement entrepreneurial de la firme nouvellement créée résulte de variables regroupées en trois catégories : les variables externes, les variables stratégiques et les variables internes. Ce comportement apparaît essentiellement comme une cause de la performance mesurée uniquement en termes économiques (rentabilité et part de marché). Pour une firme en démarrage, la performance économique conditionne sa survie ; les performances organisationnelle et sociale sont difficilement dissociables de la performance économique et ne peuvent être que difficilement isolées. À son tour, la performance peut favoriser certains comportements de la firme. Le comportement de la firme rétroagit sur les variables stratégiques qui en sont la manifestation évidente et influence de façon plus limitée les variables internes et même l'environnement par les réseaux utilisés ou mis en place par le créateur.

À l'aide de ce modèle, nous chercherons plus particulièrement à vérifier les hypothèses suivantes :

1. Le comportement de la firme nouvellement créée est déterminé par le comportement de son créateur, lequel utilisera toutes les capacités internes pour définir une stratégie pour tirer parti des occasions opportunes de l'environnement.

2. Il est possible de repérer certains comportements favorisant la performance économique.

3. Le repérage de comportements types associés à un certain niveau de performance permet de caractériser les phases du processus d'émergence.

4. En situant les entreprises dans ces phases, il devient possible de formuler un pronostic sur leur évolution future. 


\section{SCHÉMA 2}

Modèle d'émergence de la petite entreprise

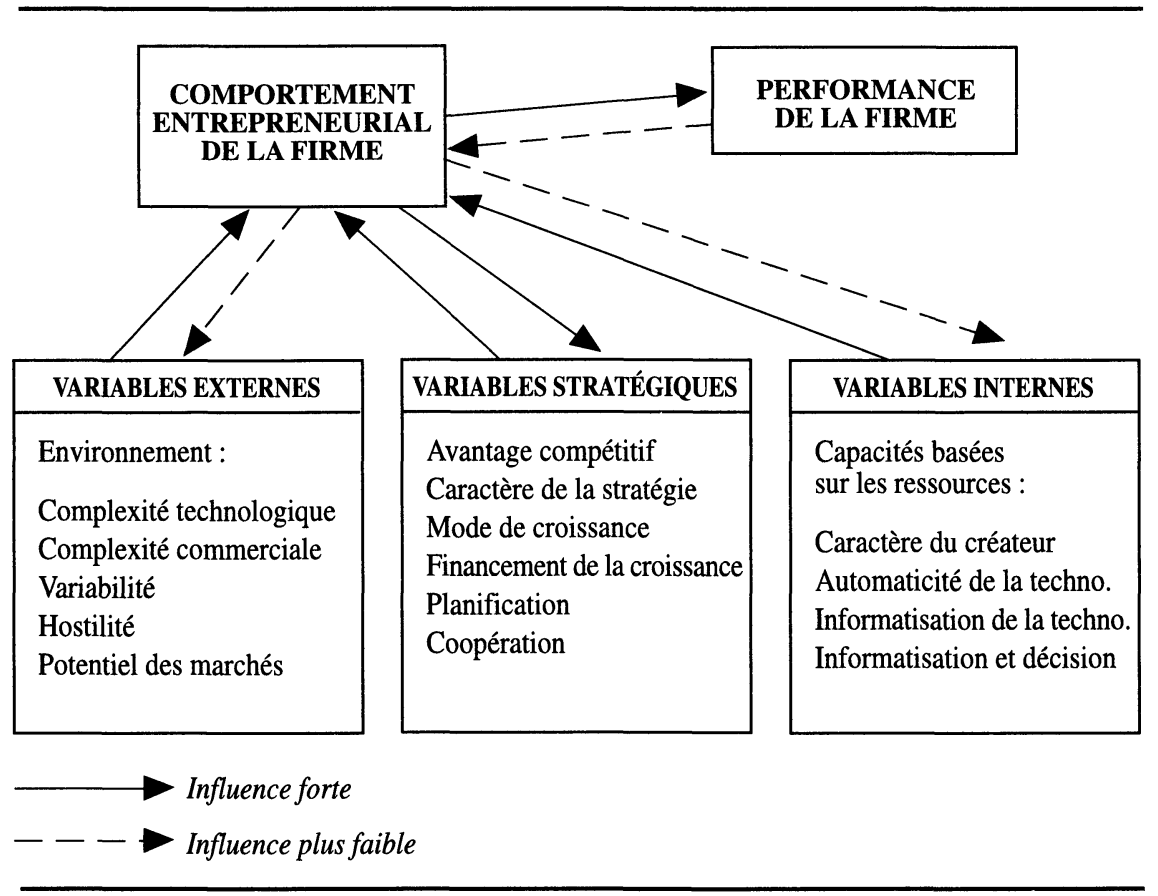

Ce modèle a été testé sur un échantillon de créations récentes.

\subsection{Caractéristiques de l'échantillon}

L'échantillon est composé de 118 entreprises créées entre le $1^{\text {er }}$ janvier 1990 et le 31 décembre 1992 dans trois départements de 1'Ouest de la France. Nous avons distingué deux catégories d'entreprises : celles issues de pépinières et celles qui ont fait cavalier seul, car on peut penser que les premières ont un comportement spécifique en raison de la sélection opérée au départ. Nous avons contacté toutes les entreprises issues des pépinières des trois départements, au nombre de 103, sur lesquelles 63 réponses exploitables ont été retenues, soit un taux de réponse de $61,2 \%$. En outre, parmi environ 11000 créations dans les trois départements répertoriées sur les fichiers des Chambres de commerce et des Chambres des métiers, 200 entreprises ont été choisies au hasard sur lesquelles 55 réponses ont pu être utilisées, soit un taux de réponse de 27,5\%.

L'enquête a été réalisée de janvier à juin 1994. Le questionnaire était composé de cinq grandes parties : 
1) caractères généraux de l'entreprise (cinq questions);

2) objectifs fixés par le créateur (14 questions);

3) caractère du créateur (cinq questions);

4) perception de l'environnement par le créateur (15 questions);

5) organisation et stratégie de l'entreprise (18 questions).

Ce questionnaire a été testé par entretien directif avec 15 créateurs, le reste des réponses ayant été obtenu par courrier avec, pour nombre d'entre elles, des précisions recueillies par entretien téléphonique.

\section{TABLEAU 1}

\section{Caractéristiques de l'échantillon}

\begin{tabular}{|c|c|c|c|c|c|}
\hline Secteur & $\begin{array}{l}\text { 1. Agro- } \\
\text { alimentaire }\end{array}$ & $\begin{array}{l}\text { 2. Industrie } \\
\text { (hors IAA) }\end{array}$ & $\begin{array}{l}\text { 3. Bâtiments } \\
\text { travaux } \\
\text { publics }\end{array}$ & 4. Services & 5. Commerce \\
\hline Effectif PP1 & 0 & 10 & 6 & 23 & 16 \\
\hline Effectif PP2 & 7 & 17 & 9 & 23 & 7 \\
\hline Effectif ens. & 7 & 27 & 15 & 46 & 23 \\
\hline Âge (1/1/94) & 1 : un an & 2: deux ans & 3 : trois ans & \multicolumn{2}{|l|}{ 4: quatre ans } \\
\hline Effectif PP1 & 2 & 22 & 15 & 16 & \\
\hline Effectif PP2 & 11 & 26 & 19 & 7 & \\
\hline Effectif ens. & 13 & 48 & 34 & 23 & \\
\hline Forme juridique & $1:$ EI & 2 : EURL & 3: SARL & 4:SA & 5 : Autres \\
\hline Effectif PP1 & 10 & 4 & 33 & 6 & 2 \\
\hline Effectif PP2 & 3 & 3 & 40 & 13 & 4 \\
\hline Effectif ens. & 13 & 7 & 73 & 19 & 6 \\
\hline Structure & $\begin{array}{l}1 \text { : Entrepre- } \\
\text { neuriale }\end{array}$ & $\begin{array}{l}2 \text { : Fonc- } \\
\text { tionnelle }\end{array}$ & \multicolumn{2}{|c|}{3 : Divisionnelle } & \\
\hline Effectif PP1 & 35 & 13 & 7 & & \\
\hline Effectif PP2 & 35 & 21 & 7 & & \\
\hline Effectif ens. & 70 & 34 & 14 & & \\
\hline$N^{\text {bre }}$ de salariés & $\begin{array}{c}1 \text { : zéro } \\
\text { sal. }\end{array}$ & $\begin{array}{l}2 \text { : un à } \\
\text { quatre sal. }\end{array}$ & $\begin{array}{c}3: \text { cinq à } \\
\quad 10 \text { sal. }\end{array}$ & $\begin{array}{l}4: 11 \text { à } \\
20 \text { sal. }\end{array}$ & $\begin{array}{l}5: 20 \text { à } \\
32 \text { sal. }\end{array}$ \\
\hline Effectif PP1 & 9 & 26 & 10 & 5 & 5 \\
\hline Effectif PP2 & 6 & 29 & 17 & 5 & 6 \\
\hline Effectif ens. & 15 & 55 & 27 & 10 & 11 \\
\hline
\end{tabular}

PP1 : Non issu de pépinières

PP2: Issu de pépinières

L'échantillon est composé essentiellement d'entreprises de très petite taille (nombre moyen de salariés égal à sept), avec 15 entreprises sans aucun salarié et 11 entreprises de 20 salariés et plus (maximum : 32 salariés). Plus de la moitié de ces petites firmes $(58 \%)$ sont des entreprises commerciales et de services, mais il 
s'agit d'un choix plus que d'une contrainte, car nous cherchons à caractériser un processus général d'émergence qui n'a pas de raisons d'être différent selon qu'elles appartiennent à l'industrie ou aux services. La forme juridique prédominante est la SARL (62\% des entreprises) puis la SA (16\%), l'entreprise individuelle (11\%) et l'EURL (6\%), les autres étant la Société en nom collectif (deux entreprises), la Société en commandite simple (2), la Société civile (2) et la Société de fait (1). Il est difficile pour des entités de taille aussi réduite d'appréhender la forme structurelle : la majorité (59\%) peut être qualifiée d'entrepreneuriale, c'est-à-dire une forme implicite préfonctionnelle, et les autres de fonctionnelles (29\%) et divisionnelles par produits (12\%).

Sur cet échantillon, nous avons mesuré les variables précitées.

\subsection{Méthode de mesure des variables}

Les variables de comportement regroupées en trois catégories (variables externes, variables stratégiques, variables internes) et les variables économiques de performance ont été mesurées sur des échelles de Likert à cinq points, par ordre d'importance croissante; chaque variable résulte souvent de l'agrégation de plusieurs variables. Cette mesure ordinale est une appréciation fournie par les créateurs ; afin de lui donner un maximum de signification, la cohérence de chaque questionnaire a été vérifiée par des questions croisées ; en cas d'incohérence, des précisions ont été demandées au créateur ; plusieurs questionnaires ont ainsi été éliminés. Remarquons que les résultats sont compatibles avec des études comparables faites précédemment (Capiez, 1988, 1992). Les variables seront présentées en catégories ordonnées d'intensité croissante (de un à cinq).

\section{Présentation et analyse des résultats}

Nous présenterons les résultats en deux temps : d'abord, une analyse unidimensionnelle permettra de repérer le comportement des entreprises nouvellement créées, puis une analyse multidimensionnelle précisera les liens entre comportement et performance et permettra de faire apparaître des comportements types pouvant constituer des étapes du processus d'émergence.

\subsection{Analyse unidimensionnelle: repérage du comportement des créations}

Trois catégories de variables caractérisent le comportement de la firme récemment créée : les variables externes ou variables d'environnement, les variables stratégiques et les variables internes basées sur les ressources de l'entreprise. La performance de ces firmes est mesurée par deux variables économiques. 


\subsubsection{Les variables externes: l'environnement}

Il est généralement admis que l'environnement présente trois dimensions (Duncan, 1972; Khandwalla, 1977) : la complexité engendrée par la diversité de l'activité de la firme (complexité technologique et complexité commerciale), la variabilité, c'est-à-dire la fréquence et l'importance des changements des facteurs environnementaux, estimés sur les plans technologique et commercial et l'hostilité correspondant aux menaces de la concurrence essentiellement. Mais l'environnement est aussi source d'occasions d'affaires, car les marchés constituent le potentiel de développement de l'entreprise récemment créée.

La complexité technologique (moyenne des scores de trois échelles portant sur les techniques de production), le niveau de connaissances scientifiques nécessaire et l'homogénéité des techniques de production dans l'entreprise est jugée plus forte par les créateurs issus de pépinières qui ont globalement un niveau scientifique plus élevé et s'implantent dans des secteurs à technologie plus sophistiquée. Mais, dans l'ensemble, la complexité technologique est concentrée sur les niveaux faible et moyen.

La complexité commerciale, appréhendée à partir de la mesure de quatre variables quantitatives (nombre de concurrents, de produits, de clients de l'entreprise, nombre de régions ou de pays où les ventes sont réalisées) transformées en classes ordonnées, ainsi que d'une variable qualitative (homogénéité des marchés de l'entreprise), apparaît globalement moyenne, ce qui semble normal pour des entreprises de petite dimension. Il n'apparaît pas de différences significatives entre les entreprises issues de pépinières et les autres.

La variabilité de l'environnement, appréciée à partir d'une série de questions portant sur la variabilité globale, la variabilité technologique (fréquence et importance des changements pour les matières et composants, le matériel, les méthodes de production, les compétences scientifiques dans le secteur) et la variabilité commerciale (fréquence et importance des changements dans les produits, les circuits de distribution et les marchés) sont jugées dans l'ensemble moyennes-fortes par les créateurs, là encore sans différence significative entre les entreprises issues de pépinières et les autres.

L'hostilité de l'environnement, limitée à l'intensité de la concurrence et dont le score a été obtenu par la moyenne des scores de deux questions, l'une sur l'importance quantitative de la concurrence, l'autre sur son organisation, apparaît moyenneforte dans l'ensemble.

Le potentiel des marchés, estimé par le créateur, est à la mesure de l'intensité de la concurrence : il correspond à une croissance plutôt forte.

C'est la stratégie qui permet au créateur de répondre à l'environnement en saisissant les occasions qu'il présente en évitant ses menaces, allant même jusqu'à l'aménager. 
TABLEAU 2

Les variables d'environnement

\begin{tabular}{lccccc}
\hline Catégories & $\mathbf{1}$ & $\mathbf{2}$ & $\mathbf{3}$ & $\mathbf{4}$ & $\mathbf{5}$ \\
\hline $\begin{array}{l}\text { Complexité } \\
\text { technologique } \\
\text { (CTL) }\end{array}$ & Très faible & Faible & Moyenne & Forte & Très forte \\
\hline Effectif PP1 & 10 & 22 & 19 & 3 & 1 \\
Effectif PP2 & 6 & 17 & 23 & 16 & 1 \\
Effectif ens. & 16 & 39 & 42 & 19 & 2 \\
$\%$ ens. & $13,6 \%$ & $33,1 \%$ & $35,6 \%$ & $16,1 \%$ & $1,7 \%$ \\
\hline
\end{tabular}

Complexité commerciale

\begin{tabular}{lccccc} 
(CCM) & Très faible & Faible & Moyenne & Forte & Très forte \\
\hline Effectif PP1 & 2 & 5 & 40 & 8 & 0 \\
Effectif PP2 & 3 & 15 & 32 & 13 & 0 \\
Effectif ens. & 5 & 20 & 72 & 21 & 0 \\
$\%$ ens. & $4,2 \%$ & $16,9 \%$ & $61,0 \%$ & $17,8 \%$ & $0,0 \%$ \\
\hline
\end{tabular}

Variabilité de

l'environnement

\begin{tabular}{lccccc} 
(VEN) & Très faible & Faible & Moyenne & Forte & Très forte \\
\hline Effectif PP1 & 1 & 9 & 28 & 14 & 3 \\
Effectif PP2 & 3 & 12 & 21 & 23 & 4 \\
Effectif ens. & 4 & 21 & 49 & 37 & 7 \\
$\%$ ens. & $3,4 \%$ & $17,8 \%$ & $41,5 \%$ & $31,4 \%$ & $5,9 \%$ \\
\hline
\end{tabular}

Intensité de la concurrence

\begin{tabular}{lccccc} 
(INC) & Très faible & Faible & Moyenne & Forte & Très forte \\
\hline Effectif PP1 & 0 & 1 & 26 & 24 & 4 \\
Effectif PP2 & 1 & 10 & 32 & 16 & 4 \\
Effectif ens. & 1 & 11 & 58 & 40 & 8 \\
$\%$ ens. & $0,8 \%$ & $9,3 \%$ & $49,2 \%$ & $33,9 \%$ & $6,8 \%$ \\
\hline $\begin{array}{l}\text { Potentiel } \\
\text { des marchés }\end{array}$ & En déclin & Stable & Croissance & Croissance & Croissance \\
(POM) & & & faible & soutenue & forte \\
\hline Effectif PP1 & 1 & 10 & 9 & 22 & 13 \\
Effectif PP2 & 0 & 7 & 16 & 29 & 11 \\
Effectif ens. & 1 & 17 & 25 & 51 & 24 \\
$\%$ ens. & $0,8 \%$ & $14,4 \%$ & $21,2 \%$ & $43,2 \%$ & $20,3 \%$ \\
\hline
\end{tabular}




\subsubsection{Les variables stratégiques}

Sans vouloir reprendre les nombreuses et diverses définitions de la stratégie, nous considérons la stratégie comme la combinaison des objectifs et des moyens. Nous retiendrons comme variables stratégiques: les objectifs du créateur, l'avantage compétitif de l'entreprise et les caractères généraux de la stratégie.

\subsubsection{Les objectifs du créateur}

Nous avons demandé aux créateurs de classer par ordre décroissant leurs objectifs à partir d'une liste établie après compilation des objectifs proposés par la littérature ; une ligne avait été réservée à d'autres objectifs possibles; cela nous a permis une qualification plus précise de certains objectifs. Les objectifs du créateur peuvent être ainsi hiérarchisés :

TABLEAU 3

Les objectifs du créateur

\begin{tabular}{|c|c|c|c|c|c|c|c|c|c|c|c|c|c|}
\hline Objectif & FLX & AUD & RMT & CCA & CPM & INF & PRE & REP & RIM & RPR & PER & CEP & RPU \\
\hline \multicolumn{14}{|l|}{ PP1 } \\
\hline Note & 4,29 & 4,47 & 4,18 & 4,11 & 4 & 3,93 & 3,97 & 3,47 & 3,55 & 3,44 & 3 & 2,51 & 2,55 \\
\hline Rang & 2 & 1 & 3 & 4 & 5 & 6 & 7 & 9 & 8 & 10 & 11 & 13 & 12 \\
\hline \multicolumn{14}{|l|}{ PP2 } \\
\hline Note & 4,56 & 4,32 & 4,3 & 4,03 & 3,75 & 3,7 & 3,75 & 3,59 & 3,49 & 3,17 & 3,1 & 2,65 & 2,3 \\
\hline Rang & 1 & 2 & 3 & 4 & 5 & 7 & 5 & 8 & 9 & 10 & 11 & 12 & 13 \\
\hline \multicolumn{14}{|l|}{ Ensemble } \\
\hline Note & 4,43 & 4,39 & 4,25 & 4,07 & 3,86 & 3,81 & 3,79 & 3,53 & 3,52 & 3,3 & 3,05 & 2,58 & 2,42 \\
\hline Rang & 1 & 2 & 3 & 4 & 5 & 6 & 7 & 8 & 9 & 10 & 11 & 12 & 13 \\
\hline
\end{tabular}

FLX $=$ Flexibilité, $\mathrm{AUD}=$ Autonomie de la décision, $\mathrm{RMT}=$ Rentabilité à moyen terme, $\mathrm{CCA}=$ Croissance du chiffre d'affaires, $\mathrm{CPM}=$ Croissance de la part de marché, $\mathrm{INF}=$ Indépendance financière, $\mathrm{PRE}=$ Préservation de l'environnement, REP = Recherche de l'épanouissement du personnel, RIM = Rentabilité immédiate, $\mathrm{RPR}=$ Recherche de prestige, $\mathrm{PER}=$ Pérennité, $\mathrm{CEF}=$ Croissance de l'effectif, RPU = Recherche de la puissance.

La flexibilité, l'autonomie de la décision et la rentabilité à moyen terme se détachent nettement. La rentabilité immédiate (difficile à obtenir dans les premières années d'activité) ne vient qu'en neuvième position. La croissance reste un impératif pour le créateur : il s'agit plus de croissance du chiffre d'affaires et de part de marché que de croissance de l'effectif, car en émergence économique, il faut avant tout atteindre une taille critique pour couvrir les frais fixes entrânés par la création. L'indépendance financière n'est pas négligée, car elle conditionne largement l'autonomie de la décision. Le souci de préserver l'environnement est présent dans les entreprises qui peuvent lui porter atteinte (seules les réponses des entreprises de l'industrie et du bâtiment ont été retenues, soit 49 sur 118). Le souci de l'épanouissement du 
personnel existe lorsque l'entreprise en emploie. Les objectifs relatifs au projet personnel du créateur viennent dans les dernières positions : recherche de prestige (rang 10) et recherche de la puissance (rang 13). La pérennité ne vient qu'au onzième rang, ce qui peut s'expliquer par la dialogique créateur-entreprise. L'horizon du créateur peut se limiter à sa période d'activité et un arrêt prématuré de son projet ne signifie pas qu'il n'a pas atteint certains de ses objectifs personnels (Bruyat, 1994).

L'atteinte des objectifs de croissance et de rentabilité suppose que l'entreprise s'appuie sur son avantage compétitif.

\subsubsection{L'avantage compétitif}

Dans son étude sur les stratégies de spécialisation, Marchesnay (1992) émet la remarque suivante : «si l'on se réfère aux stratégies génériques, on peut observer que la petite entreprise a le choix entre l'offre de produits à un coût plus bas que la concurrence, ou la différenciation de ses prestations, en proposant à côté du produit physique un ensemble de prestations immatérielles qui viendront accroître la satisfaction du client». Il apparaît que les petites entreprises récemment créées cherchent à accroître la spécificité de leur offre en utilisant leur avantage compétitif (Stoner, 1987). Aussi avons-nous demandé aux créateurs de classer leur avantage compétitif parmi les cinq proposés : le prix (NAC 1), la qualité (NAC 2), le service (NAC 3), l'effort commercial (NAC 4), la nouveauté du produit (NAC 5).

TABLEAU 4

La nature de l'avantage compétitif

\begin{tabular}{lccccc}
\hline Catégories & $\mathbf{1}$ & $\mathbf{2}$ & $\mathbf{3}$ & $\mathbf{4}$ & $\mathbf{5}$ \\
\hline $\begin{array}{l}\text { Nature de } \\
\text { l'avantage } \\
\text { compétitif (NAC) }\end{array}$ & $\begin{array}{c}\text { Prix } \\
\text { inférieur }\end{array}$ & $\begin{array}{c}\text { Qualité du } \\
\text { produit }\end{array}$ & Service & $\begin{array}{c}\text { Effort } \\
\text { commercial }\end{array}$ & $\begin{array}{c}\text { Nouveauté } \\
\text { du produit }\end{array}$ \\
\hline Rang PP1 & 5 & 2 & 1 & 2 & 4 \\
Rang PP2 & 5 & 2 & 1 & 3 & 3 \\
\hline
\end{tabular}

Les avantages privilégiés sont l'amélioration du service et la qualité. Vient ensuite la nouveauté des produits, mais une véritable nouveauté est difficile à réaliser. Quant à l'effort de promotion et de publicité, il est plus considéré comme un moyen de faire connaître son avantage compétitif qu' un avantage en soi. L'offre de produits moins chers existe, mais est moins recherchée.

\subsubsection{Les caractères généraux de la stratégie}

Nous caractériserons la stratégie par son caractère offensif ou défensif, le mode de croissance envisagé, le financement de cette croissance, l'importance de la 


\section{TABLEAU 5}

Les caractères généraux de la stratégie

\begin{tabular}{|c|c|c|c|c|c|}
\hline Catégories & 1 & 2 & 3 & 4 & 5 \\
\hline $\begin{array}{l}\text { Caractère } \\
\text { de la stratégie } \\
\text { (CSA) }\end{array}$ & $\begin{array}{l}\text { Uniquement } \\
\text { une réponse } \\
\text { à des menaces }\end{array}$ & $\begin{array}{c}\text { Plutôt } \\
\text { défensive }\end{array}$ & $\begin{array}{l}\text { Mi-offensive, } \\
\text { mi-défensive }\end{array}$ & $\begin{array}{c}\text { Plutôt } \\
\text { offensive }\end{array}$ & $\begin{array}{l}\text { Uniquement } \\
\text { une recherche } \\
\text { délibérée } \\
\text { de la croissance }\end{array}$ \\
\hline Effectif PP1 & 0 & 3 & 12 & 21 & 19 \\
\hline Effectif PP2 & 0 & 2 & 19 & 20 & 22 \\
\hline Effectif ens. & 0 & 5 & 31 & 41 & 41 \\
\hline$\%$ ens. & $0,0 \%$ & $4,2 \%$ & $26,3 \%$ & $34,7 \%$ & $34,7 \%$ \\
\hline $\begin{array}{l}\text { Mode de } \\
\text { croissance } \\
\text { envisagé (MCR) }\end{array}$ & $\begin{array}{l}\text { Croissance } \\
\text { interne } \\
\text { uniquement }\end{array}$ & $\begin{array}{l}\text { Plutôt } \\
\text { croissance } \\
\text { interne }\end{array}$ & $\begin{array}{l}\text { Croissance } \\
\text { interne } \\
\text { et externe }\end{array}$ & $\begin{array}{l}\text { Plutôt } \\
\text { croissance } \\
\text { externe }\end{array}$ & $\begin{array}{c}\text { Croissance } \\
\text { externe } \\
\text { uniquement }\end{array}$ \\
\hline Effectif PP1 & 34 & 16 & 0 & 5 & 0 \\
\hline Effectif PP2 & 45 & 11 & 0 & 5 & 2 \\
\hline Effectif ens. & 79 & 27 & 0 & 10 & 2 \\
\hline$\%$ ens. & $66,9 \%$ & $22,9 \%$ & $0,0 \%$ & $8,5 \%$ & $1,7 \%$ \\
\hline $\begin{array}{l}\text { Financement } \\
\text { de la croissance } \\
\text { (FCR) }\end{array}$ & $\begin{array}{c}\text { Auto- } \\
\text { financement } \\
\text { uniquement }\end{array}$ & $\begin{array}{l}\text { Endet- } \\
\text { tement } \\
\text { modéré }\end{array}$ & $\begin{array}{l}\text { Endet- } \\
\text { tement } \\
\text { moyen }\end{array}$ & $\begin{array}{l}\text { Endet- } \\
\text { tement } \\
\text { fort }\end{array}$ & $\begin{array}{l}\text { Politique } \\
\text { d'endet- } \\
\text { tement } \\
\text { maximum }\end{array}$ \\
\hline Effectif PP1 & 11 & 17 & 24 & 3 & 0 \\
\hline Effectif PP2 & 20 & 12 & 23 & 7 & 1 \\
\hline Effectif ens. & 31 & 29 & 47 & 10 & 1 \\
\hline$\%$ ens. & $26,3 \%$ & $24,6 \%$ & $39,8 \%$ & $8,5 \%$ & $0,8 \%$ \\
\hline Catégories & 1 & 2 & 3 & 4 & 5 \\
\hline $\begin{array}{l}\text { Importance } \\
\text { de la planification } \\
\text { (PLA) }\end{array}$ & Nulle & $\begin{array}{l}\text { Faible } \\
\text { (1 domaine, } \\
\text { durée } \\
\text { courte) }\end{array}$ & $\begin{array}{c}\text { Moyenne } \\
(2-3 \\
\text { domaines })\end{array}$ & $\begin{array}{l}\text { Forte } \\
\text { (4 do- } \\
\text { maines) }\end{array}$ & $\begin{array}{l}\text { Très forte } \\
\text { (5 domaines, } \\
\text { durée } \\
\text { longue })\end{array}$ \\
\hline Effectif PP1 & 21 & 24 & 7 & 2 & 1 \\
\hline Effectif PP2 & 10 & 16 & 23 & 11 & 3 \\
\hline Effectif ens. & 31 & 40 & 30 & 13 & 4 \\
\hline$\%$ ens. & $26,3 \%$ & $33,9 \%$ & $25,4 \%$ & $11,0 \%$ & $3,4 \%$ \\
\hline $\begin{array}{l}\text { Intensité } \\
\text { de la coopération } \\
\text { (COP) }\end{array}$ & Nulle & $\begin{array}{l}\text { Informelle } \\
\text { et limitée }\end{array}$ & $\begin{array}{l}\text { Informelle } \\
\text { large, sous- } \\
\text { traitance- } \\
\text { limitée }\end{array}$ & $\begin{array}{l}\text { Sous- } \\
\text { traitance } \\
\text { large }\end{array}$ & $\begin{array}{c}\text { Partenariat } \\
\text { large } \\
\text { (PME et GE) }\end{array}$ \\
\hline Effectif PP1 & 15 & 24 & 7 & 7 & 2 \\
\hline Effectif PP2 & 7 & 23 & 17 & 7 & 9 \\
\hline Effectif ens. & 22 & 47 & 24 & 14 & 11 \\
\hline$\%$ ens. & $18,6 \%$ & $39,8 \%$ & $20,3 \%$ & $11,9 \%$ & $9,3 \%$ \\
\hline
\end{tabular}


planification et le degré de planification. Les questions posées étant assez complexes, en particulier celles ayant trait au mode de croissance, afin d'éviter une interprétation erronée, des définitions précises ont été données. Par exemple, l'expression croissance interne est prise dans son sens classique de rachat ou de prise de participation significative dans d'autres entreprises soit concurrentes, soit actives dans d'autres domaines, afin de constituer un hypogroupe. La cohérence des réponses a été vérifiée systématiquement par un jeu de questions croisées et des précisions ont été demandées en cas d'ambiguiité.

Comme chez nombre de créateurs, la stratégie affirmée est essentiellement offensive. Le mode de croissance envisagé est essentiellement interne, quoique la croissance externe ne soit pas systématiquement rejetée ; elle est même souhaitée par $10 \%$ des créateurs. Le financement de la croissance par autofinancement $(26 \%)$ et par endettement limité (64\%) est cohérent avec la priorité accordée aux objectifs d'autonomie et d'indépendance.

La mise en œuvre de la stratégie du créateur peut s'appuyer sur une démarche planificatrice.

Pour une création, la planification correspond essentiellement à l'établissement d'un plan d'affaires, moyen de représentation de l'avenir du projet avec clarté et précision. Pour Livian et Marion (1991), le réalisme et la cohérence de ce plan peuvent constituer un bon indicateur de réussite du projet sous certaines conditions. Il a été demandé aux créateurs s'ils établissaient un plan de développement de leur affaire, quel en était l'horizon et quels domaines (finance, marketing, production, recherche-développement, personnel) faisaient l'objet d'une prévision. Nous en avons déduit un indice d'importance de la planification. Plus du quart des créateurs n'établissent pas de plan de développement, ce qui ne veut pas dire qu'ils n'ont pas de stratégie, mais que cette dernière n'est pas formalisée ou qu'elle est plus adaptative. La planification sur un an qui concerne $13 \%$ des entreprises n'est qu'un système budgétaire, souvent limité à un budget de trésorerie. Ce n'est qu'à partir d'un horizon de trois ans que l'on peut considérer que la planification devient véritablement stratégique : cela concerne $55 \%$ des entreprises de notre échantillon, avec un plan global détaillé en plans fonctionnels ; la majorité de ces firmes sont issues de pépinières. Dans l'ensemble, la planification est plus forte chez ces dernières, car les pépinières leur imposent souvent au départ l'établissement d'un plan d'affaires sur trois ans, les amenant ainsi à une démarche planificatrice.

Pour le créateur, un autre moyen de maîtriser son développement est la coopération.

Si le créateur doit s'adapter à son environnement, il cherche aussi, malgré son faible poids économique au départ, à aménager son environnement en se constituant un réseau étendu de relations extérieures grâce à une politique de coopération. Les modalités de cette coopération sont très diverses et vont des relations informelles 
(relations amicales et professionnelles, clubs de créateurs) à des relations juridiques formalisées (sous-traitance, cotraitance, franchise, GIE). Nous avons mesuré les formes de coopération selon le domaine et les partenaires, le cadre formel et informel, et nous en avons déduit un indice d'intensité de la coopération pour chaque entreprise. Les formes de coopération apparaissent très diversifiées et l'intensité correspond à une coopération largement informelle (60\% des entreprises), mais n'excluant pas la formalisation juridique ( $21 \%$ des firmes). On peut ainsi penser à une organisation en réseau concernant nombre des entreprises de notre échantillon, bien qu'il soit difficile d'en préciser l'étendue. C'est dans les entreprises issues de pépinières que la coopération est la plus intense.

La réussite de la stratégie est conditionnée par les ressources internes de la nouvelle firme.

\subsubsection{Les variables internes ou capacités basées sur les ressources}

La recherche entrepreneuriale s'est intéressée ces dernières années aux capacités basées sur les ressources de la firme, en tant que moyen de créer un avantage compétitif (Grant, 1991), en prolongeant les travaux de l'école du management stratégique (Ansoff, 1965 ; Andrews, 1980) fondés sur les théories de Schumpeter (1934) et Penrose (1959). Pour les firmes en émergence, ces capacités dépendent autant de l'aptitude du créateur à réunir ces ressources que des ressources elles-mêmes (Chandler et Hanks, 1994). Dans cet esprit, nous retiendrons comme variables internes de l'organisation nouvelle, le créateur et son attitude face à l'environnement ainsi que les ressources technologiques de la firme ; ces dernières sont plus caractéristiques de la capacité organisationnelle de la petite firme que d'une structure encore trop embryonnaire.

L'attitude du créateur à l'égard de l'environnement et du risque a été mesurée à partir des réponses à une série d'affirmations sur la réaction ou l'anticipation par rapport à la concurrence et aux évolutions de l'environnement ainsi que sur le comportement en matière de rentabilité-risque et sur la recherche d'occasions d'affaires. Les deux attitudes concordent : celle relative à l'environnement est concentrée sur le pôle proactif ( $68 \%$ sur les classes quatre et cinq), et celle relative au risque est décalée vers la recherche du risque, mais avec un centrage sur les classes trois et quatre ( $80 \%$ des réponses), ce qui laisse penser que les risques sont calculés. Ces attitudes sont à rapprocher du caractère offensif de la stratégie mis en avant par les créateurs (69\% sur les classes quatre et cinq).

La technologie peut être définie comme l'ensemble du processus de réalisation d'un bien ou d'un service. La place accordée à la technologie dans l'analyse de la gestion de la firme est fondamentale, qu'il s'agisse de stratégie (Dussauge et Ramanantsoa, 1987) ou d'organisation (Woodward, 1965). Dans une optique d'analyse de comportement stratégique et avec un échantillon comportant $58 \%$ d'entreprises 
commerciales et de services, nous avons retenu deux indicateurs d'application générale : l'automaticité et le degré d'informatisation.

L'automaticité est le degré de mécanisation et d'automatisation de l'activité, c'est-à-dire le degré d'utilisation des machines pour la réalisation des biens et des services et pour l'obtention des informations. Ce degré a été apprécié en pourcentage, pour plusieurs hypothèses allant du travail manuel à la gestion informatisée de la production, ce qui a permis d'établir cinq niveaux d'automaticité. L'informatique a d'abord été considérée comme une technologie et nous avons dénombré les activités informatisées afin de définir un indice d'informatisation de la technologie à cinq niveaux : informatisation nulle, faible (une à trois activités : comptabilité générale, facturation, paye), moyenne (quatre à cinq, avec gestion des stocks et comptabilité analytique), forte (six à huit, avec contrôle de production et contrôle de gestion) et très forte (neuf et plus, avec recherche-développement et recherche opérationnelle).

L'automaticité de la technologie se situe à un niveau faible-moyen. L'informatisation de la technologie est nulle pour $30 \%$ des entreprises et groupée sur les classes deux, trois et quatre pour le reste. Il faut remarquer que pour les entreprises commerciales et de services, l'automaticité de la technologie se confond largement avec l'automatisation des tâches administratives. L'automaticité et l'informatisation sont plus importantes dans les entreprises issues de pépinières et implantées dans des secteurs à technologie plus avancée.

Plus qu'une technologie, l'informatique, par sa puissance croissante de traitement, constitue un élément essentiel du système de décision. Aussi avons-nous demandé aux créateurs d'indiquer la fréquence d'utilisation de leur système informatique pour stocker des données qu'ils jugent indispensables pour prendre leurs décisions. Parmi les entreprises informatisées, $72 \%$ utilisent souvent ou systématiquement leur système informatique pour recueillir et traiter l'information, afin de prendre des décisions plus pertinentes, et ce, plus particulièrement chez les créateurs issus de pépinières. L'informatique, de simple outil d'aide à la gestion courante, devient un véritable instrument de décision stratégique.

C'est de la décision stratégique que dépend la performance.

\subsubsection{Les variables économiques de performance}

La mesure de la performance des firmes en émergence est particulièrement difficile : elles ont un historique limité et même les créations à succès n'atteignent souvent une rentabilité suffisante qu'après plusieurs années. Le taux de croissance des ventes n'est guère significatif les premières années, puisque ces firmes partent de zéro. La mesure de base est d'abord la survie, mais cet indicateur ne permet pas de différencier les firmes existantes. Aussi beaucoup de recherches sur les entreprises en 
TABLEAU 6

Les variables internes

\begin{tabular}{|c|c|c|c|c|c|}
\hline Classes & Un & Deux & Trois & Quatre & Cinq \\
\hline $\begin{array}{l}\text { Attitude } \\
\text { du créateur } \\
\text { face à l'envi- } \\
\text { ronnement (AEN) }\end{array}$ & Réactive & & & & Proactive \\
\hline $\begin{array}{l}\text { Effectif PP1 } \\
\text { Effectif PP2 } \\
\text { Effectif ens. } \\
\% \text { ens. }\end{array}$ & $\begin{array}{c}1 \\
1 \\
2 \\
1,7 \%\end{array}$ & $\begin{array}{c}2 \\
2 \\
4 \\
3,4 \%\end{array}$ & $\begin{array}{c}18 \\
14 \\
32 \\
27,1 \%\end{array}$ & $\begin{array}{c}20 \\
26 \\
46 \\
39,0 \%\end{array}$ & $\begin{array}{c}14 \\
20 \\
34 \\
28,8 \%\end{array}$ \\
\hline $\begin{array}{l}\text { Attitude du } \\
\text { créateur face } \\
\text { au risque (ARS) }\end{array}$ & $\begin{array}{l}\text { Craint } \\
\text { le risque }\end{array}$ & & & & $\begin{array}{l}\text { Recherche } \\
\text { le risque }\end{array}$ \\
\hline $\begin{array}{l}\text { Effectif PP1 } \\
\text { Effectif PP2 } \\
\text { Effectif ens. } \\
\% \text { ens. }\end{array}$ & $\begin{array}{c}0 \\
0 \\
0 \\
0,0 \%\end{array}$ & $\begin{array}{c}12 \\
3 \\
15 \\
12,7 \%\end{array}$ & $\begin{array}{c}17 \\
15 \\
32 \\
27,1 \%\end{array}$ & $\begin{array}{c}24 \\
38 \\
62 \\
52,5 \%\end{array}$ & $\begin{array}{c}2 \\
7 \\
9 \\
7,6 \%\end{array}$ \\
\hline $\begin{array}{l}\text { Automaticité de la } \\
\text { technologie (ATL) }\end{array}$ & Nulle & Faible & Moyenne & Forte & Très forte \\
\hline $\begin{array}{l}\text { Effectif PP1 } \\
\text { Effectif PP2 } \\
\text { Effectif ens. } \\
\% \text { ens. }\end{array}$ & $\begin{array}{c}18 \\
8 \\
26 \\
22,0 \%\end{array}$ & $\begin{array}{c}15 \\
21 \\
36 \\
30,5 \%\end{array}$ & $\begin{array}{c}18 \\
24 \\
42 \\
35,6 \%\end{array}$ & $\begin{array}{c}4 \\
9 \\
13 \\
11,0 \%\end{array}$ & $\begin{array}{c}0 \\
1 \\
1 \\
0,8 \%\end{array}$ \\
\hline $\begin{array}{l}\text { Informatisation } \\
\text { de la technologie } \\
\text { (IFT) }\end{array}$ & Nulle & $\begin{array}{c}\text { Faible } \\
(1-3 \\
\text { activités })\end{array}$ & $\begin{array}{l}\text { Moyenne } \\
\quad(4-5 \\
\text { activités })\end{array}$ & $\begin{array}{c}\text { Forte } \\
(6-8 \\
\text { activités })\end{array}$ & $\begin{array}{c}\text { Très forte } \\
(9 \text { activités } \\
\text { et }+)\end{array}$ \\
\hline $\begin{array}{l}\text { Effectif PP1 } \\
\text { Effectif PP2 } \\
\text { Effectif ens. } \\
\% \text { ens. }\end{array}$ & $\begin{array}{c}21 \\
14 \\
35 \\
29,7 \%\end{array}$ & $\begin{array}{c}11 \\
11 \\
22 \\
18,6 \%\end{array}$ & $\begin{array}{c}15 \\
24 \\
39 \\
33,1 \%\end{array}$ & $\begin{array}{c}5 \\
12 \\
17 \\
14,4 \%\end{array}$ & $\begin{array}{c}3 \\
2 \\
5 \\
4,2 \%\end{array}$ \\
\hline $\begin{array}{l}\text { Importance } \\
\text { de l'informatique } \\
\text { pour la décision } \\
\text { (IFD) }\end{array}$ & $\begin{array}{c}\text { Pas } \\
\text { d'informa- } \\
\text { tisation }\end{array}$ & $\begin{array}{l}\text { Prati- } \\
\text { quement } \\
\text { nulle }\end{array}$ & Parfois & Souvent & $\begin{array}{l}\text { Systémati- } \\
\text { quement }\end{array}$ \\
\hline $\begin{array}{l}\text { Effectif PP1 } \\
\text { Effectif PP2 } \\
\text { Effectif ens. } \\
\% \text { ens. }\end{array}$ & $\begin{array}{c}21 \\
14 \\
35 \\
29,7 \%\end{array}$ & $\begin{array}{c}2 \\
3 \\
5 \\
4,2 \%\end{array}$ & $\begin{array}{c}9 \\
9 \\
18 \\
15,3 \%\end{array}$ & $\begin{array}{c}10 \\
17 \\
27 \\
22,9 \%\end{array}$ & $\begin{array}{c}13 \\
20 \\
33 \\
28,0 \%\end{array}$ \\
\hline
\end{tabular}


démarrage utilisent-elles des instruments de mesure économique comme la rentabilité ou la part de marché. McDougall et DeNisi (1992) considèrent la rentabilité économique de la firme (ROI) et la croissance de la part de marché ; Sandberg et Hofer (1987), la survie associée à la rentabilité des capitaux propres (ROE); Covin et Slevin $(1989,1991)$, un indicateur complexe mis au point par Gupta et Govindarajan (1984) pondérant la satisfaction retirée de différents critères (niveau et taux de croissance des ventes, cash-flow, ROE, ROI, profitabilité) par l'importance accordée à chaque critère.

Nous avons privilégié deux indicateurs simples: la rentabilité économique et l'importance de la part de marché, en raison de l'interaction fréquente entre l'obtention d'une taille critique et la rentabilité. Il s'agit ici de la rentabilité économique du dernier exercice (Résultat d'exploitation + BFRE / Immobilisations nettes d'exploitation), ainsi que d'une estimation par le créateur de sa part de marché actuelle, à partir d'un indicateur composite : la part de marché «servi » $(\mathrm{de}<5 \%$ à $>40 \%)$ et la part de marché relative en multiple du concurrent le plus important (de $\times<0,5$ à $\times>3$ ). Pour éviter toute ambiguiité, des définitions précises ont été données pour les différents concepts de rentabilité et de part de marché et des compléments d'information ont été demandés lorsque cela était nécessaire.

\section{TABLEAU 7}

Les variables économiques de performance

\begin{tabular}{|c|c|c|c|c|c|}
\hline Classes & Un & Deux & Trois & Quatre & Cinq \\
\hline $\begin{array}{l}\text { R. expl. } \\
\text { /INE+BFRE = } \\
\text { Rentabilité } \\
\text { économique (REC) }\end{array}$ & $<0 \%$ & $0 \%<-<5 \%$ & $5 \%<-<10 \%$ & $10 \%<-<20 \%$ & $>20 \%$ \\
\hline $\begin{array}{l}\text { Effectif PP1 } \\
\text { Effectif PP2 } \\
\text { Effectif ens. } \\
\% \text { ens. }\end{array}$ & $\begin{array}{c}4 \\
3 \\
7 \\
5,9 \%\end{array}$ & $\begin{array}{c}30 \\
20 \\
50 \\
42,4 \%\end{array}$ & $\begin{array}{c}16 \\
24 \\
40 \\
33,9 \%\end{array}$ & $\begin{array}{c}5 \\
14 \\
19 \\
16,1 \%\end{array}$ & $\begin{array}{c}0 \\
2 \\
2 \\
1,7 \%\end{array}$ \\
\hline $\begin{array}{l}\text { Importance de la } \\
\text { part de marché } \\
\text { (IPM) }\end{array}$ & $\begin{array}{l}<5 \% \text { et } \\
x<0,5\end{array}$ & $\begin{array}{c}5 \%<-<10 \% \\
\text { et } 0,5<x<1\end{array}$ & $\begin{array}{c}10 \%<-<20 \% \\
\text { et } 1<x<1,5\end{array}$ & $\begin{array}{c}20 \%<-<40 \% \\
\text { et } 1,5<x<3\end{array}$ & $\begin{array}{c}>40 \% \text { et } \\
\quad x>3\end{array}$ \\
\hline $\begin{array}{l}\text { Effectif PP1 } \\
\text { Effectif PP2 } \\
\text { Effectif ens. } \\
\text { \% ens. }\end{array}$ & $\begin{array}{c}6 \\
2 \\
8 \\
6,8 \%\end{array}$ & $\begin{array}{c}18 \\
10 \\
28 \\
23,7 \%\end{array}$ & $\begin{array}{c}22 \\
26 \\
48 \\
40,7 \%\end{array}$ & $\begin{array}{c}9 \\
22 \\
31 \\
26,3 \%\end{array}$ & $\begin{array}{c}0 \\
3 \\
3 \\
2,5 \%\end{array}$ \\
\hline
\end{tabular}

Si $48 \%$ des entreprises ont une rentabilité économique faible (ne dépassant pas $5 \%$ ), $52 \%$ réalisent une performance assez satisfaisante ; les meilleures rentabilités se rencontrent surtout dans les entreprises issues de pépinières et les plus 
faibles, dans les entreprises âgées de un ou deux ans, ce qui n'est guère étonnant. Par contre, la part de marché actuelle est jugée plus satisfaisante dans l'ensemble (moyenne et forte pour $67 \%$ des entreprises), ce qui peut s'expliquer par la dimension limitée des segments de marché visés par les créateurs.

Il faut maintenant étudier les liens entre ces différentes variables, afin de mettre en évidence les causes de l'émergence ainsi que le processus d'émergence.

\subsection{Analyse multidimensionnelle : le processus d'émergence}

Nous procéderons en deux temps :

- d'abord une analyse des correspondances multiples (ACM) recherchera des causes d'émergence (analyse contingente);

- puis une classification ascendante hiérarchique (CAH) des entreprises permettra d'obtenir des groupes homogènes caractérisés par un comportement stratégique particulier correspondant à des phases du processus d'émergence (analyse processuelle).

\subsubsection{Analyse contingente de l'émergence}

C'est le degré de performance obtenu qui caractérise l'émergence et qui trouve son origine dans le comportement entrepreneurial de l'entreprise. L'ACM, en faisant ressortir les relations les plus significatives entre les diverses variables étudiées, fait apparaître des causes de l'émergence. Mais, auparavant, nous avons recherché les liaisons les plus significatives entre les variables à l'aide d'un test du khi carré.

\subsubsection{Khi carré et liaisons significatives}

Le test du khi carré indique des liaisons significatives

- entre variables environnementales : complexité commerciale, intensité de la concurrence et variabilité de l'environnement ;

- entre variables environnementales, variables de ressources internes et variables stratégiques : complexité technologique avec automatisation et informatisation de la technologie (liées entre elles), ainsi qu'avec planification et coopération ;

- entre variables de ressources internes, variables stratégiques et variables de performances : automatisation et informatisation de la technologie, informatisation de la décision, coopération avec rentabilité économique et importance de la part de marché (liées entre elles).

Ces relations vont être précisées par l'ACM. 


\section{TABLEAU 8}

Khi carré entre variables significatives

\begin{tabular}{|c|c|c|c|c|c|c|c|c|c|c|c|c|}
\hline & \multicolumn{4}{|c|}{ Environnement } & \multicolumn{2}{|c|}{ Stratégie } & \multicolumn{4}{|c|}{ Ressources internes } & \multicolumn{2}{|c|}{ Performance } \\
\hline & $\mathrm{CCM}$ & CTL & VEN & INC & PLA & $\mathrm{COP}$ & AEN & ATL & IFT & IFD & REC & IPM \\
\hline \multicolumn{13}{|l|}{$\mathrm{CCM}$} \\
\hline CTL & NS & & & & & & & & & & & \\
\hline VEN & $\begin{array}{c}39 * * * \\
(9)\end{array}$ & NS & & & & & & & & & & \\
\hline INC & $\begin{array}{c}27 * * * \\
(9)\end{array}$ & NS & NS & & & & & & & & & \\
\hline PLA & NS & $\begin{array}{r}19 * \\
(9)\end{array}$ & NS & $\begin{array}{c}29 * * * \\
(9)\end{array}$ & & & & & & & & \\
\hline $\mathrm{COP}$ & NS & $\begin{array}{c}26^{* * *} \\
(12)\end{array}$ & NS & NS & $\begin{array}{l}25^{*} \\
(12)\end{array}$ & & & & & & & \\
\hline AEN & NS & NS & $\begin{array}{l}20^{*} \\
(9)\end{array}$ & $\begin{array}{c}22^{* *} \\
(9)\end{array}$ & NS & NS & & & & & & \\
\hline ATL & NS & $\begin{array}{c}67 * * * \\
(9)\end{array}$ & NS & NS & NS & $\begin{array}{c}34 * * * \\
(12)\end{array}$ & NS & & & & & \\
\hline IFT & NS & $\begin{array}{c}75^{* * *} \\
(9)\end{array}$ & NS & NS & NS & $\begin{array}{c}30 * * * \\
(12)\end{array}$ & NS & $\begin{array}{c}82 * * * \\
(9)\end{array}$ & & & & \\
\hline IFD & $\begin{array}{l}20^{*} \\
(9)\end{array}$ & $\begin{array}{c}72 * * * \\
(9)\end{array}$ & NS & NS & NS & $\begin{array}{l}24^{*} \\
(12)\end{array}$ & $\begin{array}{l}19^{*} \\
(9)\end{array}$ & $\begin{array}{c}78 * * * \\
(9)\end{array}$ & $\begin{array}{c}83^{* * *} \\
(9)\end{array}$ & & & \\
\hline REC & NS & $\begin{array}{c}37 * * * \\
(9)\end{array}$ & NS & NS & NS & $\begin{array}{c}43 * * * \\
(12)\end{array}$ & $\begin{array}{l}21^{*} \\
(9)\end{array}$ & $\begin{array}{c}64 * * * \\
(9)\end{array}$ & $\begin{array}{c}60 * * * \\
(9)\end{array}$ & $\begin{array}{c}55^{* * *} \\
(9)\end{array}$ & & \\
\hline IPM & NS & $\begin{array}{l}21^{*} \\
(9)\end{array}$ & NS & NS & NS & NS & NS & $\begin{array}{c}29 * * * \\
(9)\end{array}$ & $\begin{array}{c}33 * * * \\
(9)\end{array}$ & $\begin{array}{c}30 * * * \\
(9)\end{array}$ & $\begin{array}{c}79 * * * \\
(9)\end{array}$ & \\
\hline
\end{tabular}

Entre parenthèses figure le nombre de degrés de liberté.

NS : non significatif.

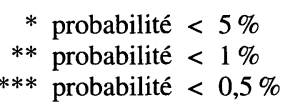

$\mathrm{CCM}$ : complexité commerciale

CTL: complexité technologique

VEN : variabilité de l'environnement

INC : intensité de la concurrence

PLA : planification

COP : coopération
AEN : attitude face à l'environnement

ATL : automaticité de la technologie

IFT : informatisation de la technologie

IFT : informatisation pour la décision

REC : rentabilité économique

IPM : importance de la part de marché 


\subsubsection{Analyse des correspondances multiples; l'origine de la performance}

L'ACM porte sur 18 variables : cinq relatives à l'environnement (CTL, CCM, VEN, INC, POM), six relatives à la stratégie (NAC, CSA, MCR, FCR, PLA, COP), cinq relatives aux capacités basées sur les ressources (AEN, ARS, ATL, IFT, IFD) et les deux variables de performance (REC, IPM), avec 85 modalités.

Les six premiers axes expliquent respectivement $10,35 \%, 7,74 \%, 4,87 \%$, $4,52 \%, 4,32 \%$ et $4,22 \%$ de l'inertie, soit un cumul de $36,02 \%$, ce qui est acceptable compte tenu du nombre de variables et de modalités. Nous analyserons les deux premiers axes en raison de leur fort pouvoir discriminant. Ils mettent en évidence l'origine de la performance.

SCHÉMA 3

L'origine de la performance

AXE 2 : coopération, planification, performance

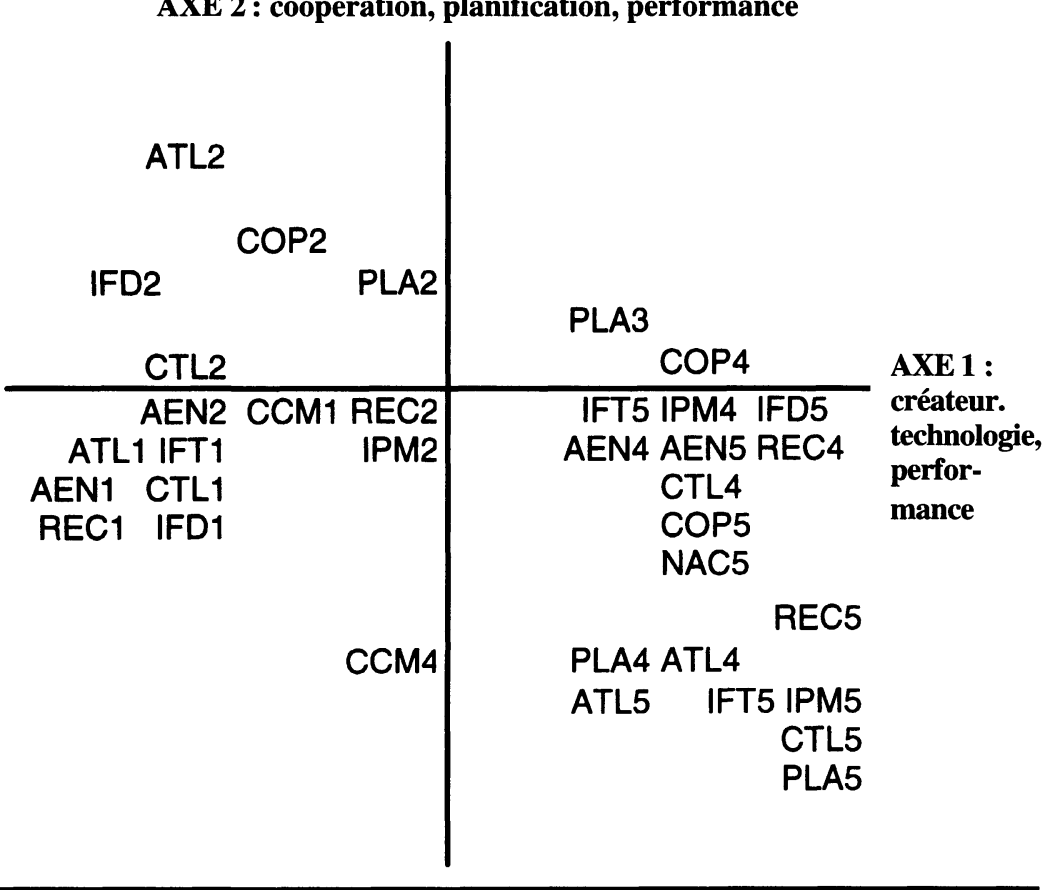

Le premier axe peut être qualifié de «créateur, technologie, performance ». Sur la partie positive figurent les entreprises à créateur proactif (AEN 5), fortement informatisées et automatisées (ATL 4, IFT 4, IFD 5), avec pour certaines un produit 
innovant (NAC 5), évoluant dans un environnement complexe (CTL 5) et à performances élevées (REC 4, REC 5, IPM 5), alors que la partie négative rassemble des firmes à créateur plus réactif ( $\mathrm{AEN} 1, \mathrm{AEN} 2)$, à automatisation et informatisation inexistantes (ATL 1, IFT 1), dans un environnement simple (CTL 1, CCM 1), avec des performances limitées (REC 1, REC 2, IPM 1, IPM 2).

Le deuxième axe fait apparaître l'influence de la planification et de la coopération sur la performance. Il semble que les firmes qui coopèrent peu (COP 2) et ne planifient guère leur développement évoluent dans un environnement technologique peu complexe (CTL 2) [partie positive de l'axe] et que, lorsque la complexité s'accroît (CCM 4, CTL 4, CTL 5), la coopération s'élargit (COP 5), la planification devient systématique (PLA 4, PLA 5) et les performances s'améliorent (REC 4, IPM 5) [partie négative de l'axe].

L'ACM confirme et approfondit les relations qu'a fait ressortir le test du khi carré. Il apparaît ainsi que, face à un environnement complexe (en matière de technologie principalement, mais aussi en matière commerciale), le caractère proactif du créateur l'amène à automatiser et à informatiser la technologie de son entreprise et à utiliser systématiquement l'informatique dans sa décision, afin de saisir au mieux les circonstances opportunes offertes par cet environnement et de le maîtriser le plus possible. La planification du développement de l'entreprise et une coopération avec tous les acteurs influents vont de pair avec une complexité croissante. Un tel comportement stratégique favorise la rentabilité et renforce le pouvoir de marché, ce qui permet l'émergence.

Mais l'émergence est avant tout un processus que l'on peut appréhender en repérant divers comportements stratégiques qui correspondent à des phases bien précises de ce processus.

\subsubsection{Analyse processuelle de l'émergence}

Une CAH permet d'établir une typologie des entreprises caractérisées par un certain comportement stratégique. Elle distingue cinq classes d'entreprises que nous avons appelées en fonction de leur comportement : les « incertaines », les « valeurs sûres », les «dynamiques », les «tranquilles » et les « innovatrices ».

Classe 1 : les « incertaines ». Il s'agit de 38 entreprises plutôt jeunes, de petite taille, non ou peu informatisées, souvent commerciales ou de services, dont $29 \%$ sont issues de pépinières. Leur rentabilité est faible et la possibilité d'échec reste grande pour nombre d'entre elles.

Classe 2 : les « valeurs sûres ». Cette classe regroupe 33 entreprises plus âgées, le plus souvent industrielles, qui ont su tirer parti d'un environnement complexe en s'insérant dans un créneau porteur, grâce à une technologie évoluée basée sur l'automatisation et l'informatisation ainsi que, pour certaines, à un réseau étendu de 
coopération et à une capacité à planifier systématiquement leur développement. Cela se traduit par une bonne rentabilité et une part importante du domaine d'activité dans lequel elles ont su s'implanter. Elles proviennent à $55 \%$ de pépinières.

Classe 3 : les «dynamiques ». Cette classe est composée de 10 entreprises jeunes (dont huit issues de pépinières), dans les secteurs du bâtiment et de l'industrie, avec à leur tête un dirigeant proactif prenant des risques pour développer son organisation dans un environnement jugé complexe et évolutif. Elles sont informatisées mais n'utilisent pas forcément toutes les possibilités de cet outil. Leurs résultats sont assez satisfaisants surtout au regard de la part de marché. Leur avenir repose sur la capacité du créateur à adapter leur technologie pour répondre aux exigences d'une demande évolutive.

Classe 4 : les «tranquilles ». Ces 27 entreprises appartenant à des secteurs variés et dont $59 \%$ proviennent de pépinières ont un créateur plus réactif. Elles réalisent leur activité dans un environnement commercial et technologique relativement simple et obtiennent des performances limitées en part de marché et moyennes en rentabilité.

Classe 5 : les « innovatrices ». Ces 10 entreprises industrielles, toutes issues de pépinières, réalisent leur croissance grâce à un produit nouveau fabriqué à l'aide de techniques modernes. Les créateurs utilisent les possibilités de l'informatique aussi bien pour la fabrication que pour la planification de leur croissance et développent souvent un véritable partenariat avec des groupes industriels. Leurs performances sont excellentes, aussi bien sur le plan de la part de marché que sur celui de la rentabilité.

On peut rapprocher ces typologies d'entreprises des typologies d'entrepreneurs comme celles proposées, entre autres, par Miles et Snow selon les conditions de l'innovation (voir Julien et Marchesnay, 1996, p. 56-57) ; certes, le créateur des «innovatrices» présente des traits communs avec le «prospecteur»ou l' « innovateur », mais la dimension est autre puisqu'elle englobe toutes les capacités basées sur les ressources internes. Comme le remarquent Covin et Slevin (1991, p. 20), « les organisations comme les individus peuvent créer de la valeur pour la société par une combinaison réfléchie et productive de ressources ; limiter la discussion du processus entrepreneurial aux individus est indûment restrictif ».

La CAH confirme ainsi le rôle essentiel de la technologie, associé à la planification et à la coopération dans la réussite de l'émergence. Nous pouvons maintenant discuter l'ensemble de nos résultats.

\section{Discussion et conclusion}

Cette étude recherche, d'une part, une relation de causalité du comportement entrepreneurial de la firme, performance permettant l'émergence, et, d'autre part, une caractérisation du processus d'émergence. Les résultats obtenus vont dans ce sens. 
En matière de causalité, l'analyse des correspondances multiples entre variables fait apparaître :

- un axe «créateur, technologie, performance» validant l'hypothèse 1 de notre modèle : le comportement du créateur (caractère plus ou moins proactif), utilisant toutes les ressources de l'organisation qu'il a mise en place, détermine un véritable comportement entrepreneurial de la firme. Ce comportement se caractérise par une stratégie permettant d'exploiter au maximum les occasions offertes par l'environnement. Plus l'environnement se révèle complexe, plus il utilise les ressources technologiques aussi bien pour l'établissement d'un système d'information pertinent que pour la production. À ce comportement est associée la performance économique, et ce, d'autant plus qu'est proposée une véritable innovation en matière de biens ou services.

- un axe « coopération, planification, performance » qui valide l'hypothèse 2: le comportement de la firme, fondé sur une coopération étendue avec les acteurs influents de l'environnement et sur une organisation privilégiant l'utilisation de l'information pour la décision, améliore la performance, aussi bien en termes de rentabilité que de part de marché. Il serait intéressant d'approfondir, plus que nous n'avons pu le faire dans cette enquête, l'étendue et les caractéristiques du réseau d'alliances que les petites firmes ont su constituer.

Nous pouvons déduire de ces résultats le paradigme de l'émergence (EMG):

$$
\mathrm{EMG}=\mathrm{f}(\mathrm{E}-\mathrm{O}, \mathrm{S}, \mathrm{EV}, \mathrm{E}-\mathrm{O} \times \mathrm{S} \times \mathrm{EV})
$$

lequel fait ressortir que plus que les variables elles-mêmes (entrepreneur et organisation, stratégie, environnement), c'est leur interaction qui explique l'émergence.

Mais l'émergence ne peut se limiter à une simple approche causale. Le processus de l'émergence doit être mis en évidence. C'est ce qu'a permis la classification hiérarchique des firmes de notre échantillon. La classification opère des regroupements caractérisés par certains types de comportement que l'on peut considérer comme caractéristiques des phases du processus d'émergence (voir schéma 4).

À l'issue du démarrage, certaines entreprises courent à l'échec ou ont un avenir incertain, soit qu'elles n'ont pas su faire évoluer leur projet ou qu'elles manquent de moyens, ou les deux raisons à la fois. Il s'agit ici de celles que nous avons qualifiées d' «incertaines ». On retrouve dans cette catégorie les firmes n'ayant aucun employé ; dans le meilleur des cas (la survie), le créateur doit plutôt être considéré comme un travailleur autonome. 


\section{SCHÉMA 4}

Processus d'émergence et comportements stratégiques

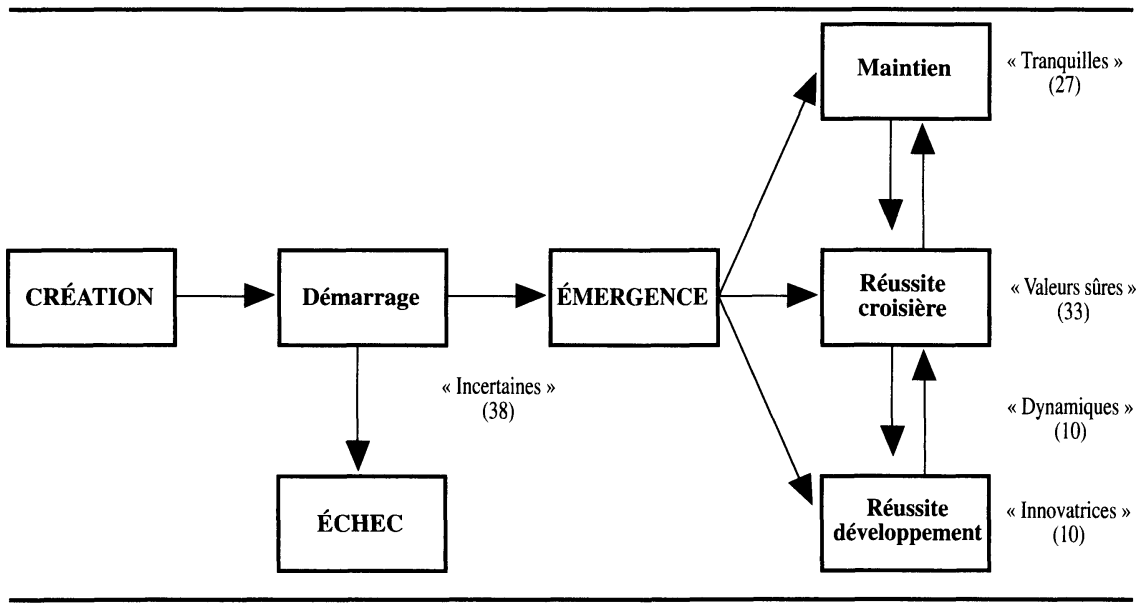

Les autres entreprises ont réalisé leur émergence, mais les situations sont variées. Trois catégories apparaissent.

Les entreprises en stade de maintien: elles ont dépassé leur taille critique permettant d'absorber les coûts fixes et atteint une dimension, jugée optimale par le créateur qui souhaite garder son autonomie de décision et son indépendance financière, avec une performance économique considérée comme satisfaisante ; elles ne connaîtront plus guère de croissance et nous les avons appelées les « tranquilles».

Les entreprises en stade de réussite-croisière : elles ont atteint une dimension plus importante que les précédentes avec de meilleures performances; elles ont un pouvoir de marché certain dans un créneau bien sûr limité, avec un niveau technologique élevé ; nous les avons dénommées les "valeurs sûres ».

Les entreprises en stade de réussite-développement : celles-ci sont susceptibles de devenir des entreprises plus importantes, si elles continuent à s'adapter en permanence à l'environnement et à en exploiter les meilleures occasions d'affaires. Celles que nous avons appelées « innovatrices » répondent à ces conditions : elles réalisent une véritable innovation de produit à l'aide d'une technique moderne et obtiennent d'excellentes performances tant pour la croissance que pour la rentabilité. Celles que nous avons qualifiées de «dynamiques » peuvent entrer dans cette catégorie en raison de la pugnacité de leur créateur face à un environnement complexe et mouvant; mais les risques sont à la hauteur de l'espoir placé dans le dirigeant qui doit savoir adapter une technologie encore embryonnaire à une demande évolutive. 
Ces stades caractérisent-ils le processus d'émergence ? On peut le penser si l'on considère que les entreprises vont cheminer le long du sentier qu'ils tracent. Mais le cheminement peut être complexe. Si certaines courent plus ou moins vite à l'échec, celles qui ont émergé peuvent s'engager dans une réussite-croisière qui peut être longue et revenir à un stade de maintien ou au contraire évoluer vers une réussite-développement ; à l'inverse, une réussite-développement peut tourner court et revenir à une réussite-croisière, voire au simple maintien. L'hypothèse 3 peut être considérée comme validée : le processus d'émergence existe et se concrétise par un cheminement en plusieurs étapes ; mais comme l'a déjà montré une étude précédente (Capiez, 1992), des pauses et des retours en arrière sont fréquents. L'émergence apparaît moins comme un lent et continu processus de type darwinien que comme un processus «ponctuationniste» ou «saltationniste», constitué de périodes relativement stables et souvent longues, ponctuées de périodes brèves de bouleversement permettant de sauter à une nouvelle phase. Même si nous nous référons à un concept biologique de cycle de vie, nous nous éloignons du schéma simpliste de l'entreprise qui naît petite et devient graduellement grande sous peine de disparaître; au contraire, nous vérifions que «l'évolution des entreprises est rarement linéaire... la plupart des petites entreprises demeurent petites, tout en évoluant à des rythmes souvent différents les unes des autres et tout en étant très performantes » (Julien et Marchesnay, 1996, p. 74). Cependant, on ne peut nier que la possibilité de grandir existe, même si la probabilité en est faible; dans l'enquête de l'INSEE citée en introduction, $0,2 \%$ des créations ont dépassé la taille de 50 salariés au bout de cinq ans d'existence.

Quant à l'hypothèse 4, la validation est plus discutable. Si nous pouvons placer les firmes de l'échantillon dans des phases significatives du processus d'émergence, peut-on formuler un pronostic sur l'étendue de la réussite d'une petite entreprise en émergence ? Le modèle proposé permet juste de repérer les firmes qui ont le plus de chances de se développer en réalisant un saut décisif, ici, les «innovatrices », qui paraissent en plein processus de réussite-développement, et, dans une moindre mesure, les «dynamiques », à condition qu'elles adaptent leurs moyens à leurs ambitions. Pour aller plus loin, il faudrait que la recherche en entrepreneuriat construise des indicateurs de réussite à partir d'éléments financiers, stratégiques, organisationnels et de capital humain.

Mais, tel quel, ce modèle peut fournir à des organismes de capital de risque publics ou privés des éléments de sélection au regard de petites entreprises susceptibles de réaliser un développement notoire.

Si l'on considère l'attitude entrepreneuriale de la petite firme comme un phénomène comportemental, il peut être géré. Les composants sous-jacents de ce comportement, prise de risque, proactivité et innovation, peuvent être encouragés et dotés de moyens. 
Remarquons, pour terminer, le rôle positif des pépinières dans l'émergence des créations. La sélection des dossiers opérée, l'aide apportée à la mise au point d'un projet viable et l'établissement d'un plan d'affaires semblent porter leurs fruits puisque sont issues de pépinières toutes les « innovatrices », $80 \%$ des « dynamiques », $55 \%$ des « valeurs sûres » et $59 \%$ des «tranquilles » contre $29 \%$ seulement des « incertaines ».

\section{Bibliographie}

ALDRICH, H., B. RosEN et W. WoODWARD (1986), « Social behavior and entrepreneurial networks », Frontiers of Entrepreneurship Research, Babson College, p. 239-240.

ALDRICH, H., B. RosEN et W. WOODWARD (1987), «The impact of social networks on business foundings and profit : a longitudinal study », Frontiers of Entrepreneurship Research, Babson College, p. 154-168.

ANDREWS, K. (1980), The Concept of Corporate Strategy, Homewood, Ill., Richard D. Irwin.

ANSOFF, H.I. (1965), Corporate Strategy, New York, McGraw-Hill.

BAMFORD, C., T. DEAN et P. MCDOUGALL (1996), «Initial founding conditions and new firm performance : a longitudinal study integrating predictions from multiple perspectives », Frontiers of Entrepreneurship Research, Babson College, p. 465-479.

BAMFORD, C., T. DEAN et P. MCDougall (1997), «Initial strategies and new venture growth : an examination of the effectiveness of broad vs. narrow breadth strategies », Frontiers of Entrepreneurship Research, Babson College, p. 375-389.

BEGLEY, T. et D. BOYD (1986), « Psychological characteristics associated with entrepreneurial performance », Frontiers of Entrepreneurship Research, Babson College, p. 146-165.

BIRLEY, S. (1985), «The role of networks in entrepreneurial process », Frontiers of Entrepreneurship Research, Babson College, p. 146-165.

BonNAUD, J. (1994), «La création d'entreprise, source de renouvellement du tissu des PME », Économie et Statistique, ${ }^{\text {os }} 271-272$, p. 25-36.

BRUYAT, C. (1994), «Contribution épistémologique au domaine de l'entrepreneurship », Revue Française de Gestion, n ${ }^{\circ} 101$, p. 87-99.

BYGRAVE, W. (1989), «The entrepreneurial paradigm : a philosophical look at its research methodologies », Entrepreneurship Theory and Practice, vol. 14, n ${ }^{\circ}$ 1, Baylor University, p. 7-26.

Bygrave, W. et C. Hofer (1990), «Theorizing about entrepreneurship ", Entrepreneurship Theory and Practice, vol. 16, $\mathrm{n}^{\circ}$ 2, Baylor University, p. 13-22.

CAMPBELl, C. (1992), «A decision theory model for entrepreneurial acts», Entrepreneurship Theory and Practice, vol. 17, $\mathrm{n}^{\circ}$ 1, Baylor University, p. 21-27.

CAPIEZ, A. (1988), «Conditions et modalités d'émergence des TPE et PE : l'exemple du Maine-et-Loire (France) », Revue Internationale PME, vol. 1, $\mathrm{n}^{\circ}$ 2, p. 128-155.

CAPIEZ, A. (1992), «Les chances de succès des petites entreprises : vers un diagnostic d'émergence », Revue Internationale PME, vol. 5, nº 2, p. 104-132. 
CARland, J., F. HoY et J. A. CARland (1988), «Who is an entrepreneur? Is a question worth asking ? », American Journal of Small Business, printemps 1988, p. 21-27.

CARrIÈRE, J. (1990), « La vision stratégique en contexte de PME, cadre théorique et étude empirique », Revue Internationale PME, vol. 3, $\mathrm{n}^{\text {os }} 3-4$, p. 301-325.

CARTER, N., T. STEARns, P. Reynolds et B. Miller (1994), «New venture strategies : theory development with an empirical base », Strategic Management Journal, vol. 15, $\mathrm{n}^{\circ} 1$, p. 21-41.

Castrogiovanni, G. (1996), «Pre-startup planning and the survival of new small businesses : theoretical linkages », Journal of Management, vol. 22, $\mathrm{n}^{\circ}$ 6, p. 801822.

Chaganti, R., R. Chaganti et V. Marajan (1988), « Profitable small business strategies under different types of competition », Entrepreneurship Theory and Practice, vol. 13, $\mathrm{n}^{\circ}$ 3, Baylor University, p. 21-35.

ChANDLER, G. et S. HANKS (1994), « Market attractiveness, ressources-based capabilities, venture strategies, and venture performance », Journal of Business Venturing, vol. 9, no 4, p. 331-349.

CoOper, A., W. Dunkelberg et C. Woo (1988), «Survival and failure : a longitudinal study », Frontiers of Entrepreneurship Research, Babson College, p. 225-237.

Covin, J. et D. SLEVIN (1988a), « New venture competition strategy : an industrial life cycle analysis », Frontiers of Entrepreneurship Research, Babson College, p. 446-460.

Covin, J. et D. SLEVIN (1988b), «Empirical relationship among strategic posture environmental context variables, and new venture performance », Frontiers of Entrepreneurship Research, Babson College, p. 446-460.

COVIN, J. et D. SLEVIN (1989), « Strategic management of small firms in hostile and benign environments », Journal of Management Studies, vol. 25, nº 3, p. 217-234.

COVIN, J. et D. SLEVIN (1991), « A conceptual model of entrepreneurship as firm behavior », Entrepreneurship Theory and Practice, Baylor University, vol. 16, $\mathrm{n}^{\circ}$ 1, p. 7-25.

D'AMBOISE, G. et A. BAKANIBONA (1990), «La planification dans les PME : une synthèse des résultats empiriques. Conclusions et recommandations », Revue Internationale $P M E$, vol. 3, no 2 , p. 147-166.

DubINI, P et H. AlDRICH (1991), «Personal and extended networks are central to the entrepreneurial process », Journal of Business Venturing, vol. 6, $\mathrm{n}^{\circ}$ 5, p. 305-313.

DUNCAN, R. (1972), «Characteristics of organizational environment and perceived environmental uncertainty », Administrative Science Quarterly, vol. 17, p. 313-327.

Dussauge, P. et B. RAMANANTSOA (1987), Technologie et stratégie de l'information, Paris, McGraw-Hill, 248 p.

GARTNER, W. (1985), «A conceptual framework for describing the phenomenon of new venture creation », Academy of Management Review, vol. 10, $\mathrm{n}^{\circ} 4$, p. 696-706.

GARTNER, W. (1988), «Who is an entrepreneur? Is it the wrong question? », American Journal of Small Business Venturing, printemps, p. 11-32.

GARTNER, W. (1993), «Words lead to deeds : towards an organizational emergence vocabulary », Journal of Business Venturing, vol. 8, $\mathrm{n}^{\circ} 3$, p. 231-239. 
GARTNER, W., B. BIRD et J. STARR (1992), « Acting as if : differenciating entrepreneurial from organizational behavior», Entrepreneurship Theory and Practice, Baylor University, vol. $16, \mathrm{n}^{\circ} 3, \mathrm{p} .13-31$.

GRANT, R. (1991), «A resource-based theory of competitive advantage : implications for strategy formulation », California Management Review, printemps, p. 114-135.

GREENBERGER, D. et D. SEXTON (1988), «An interactive model of new venture initiation», Journal of Small Business Management, vol. 26, $\mathrm{n}^{\circ}$ 3, p. 1-7.

GREPME (1995), Les PME: Bilan et perspectives, Cap-Rouge, Presses Inter Universitaires ; Paris, Economica.

GuPTA, A. et V. GOVINDARAJAN (1984), « Business unit strategy managerial characteristics, and business unit effectiveness at strategy implementation », Academy of Management Journal, vol. 27, no 3, p. 25-41.

Guth, W., A. Kumaraswany et M. MCERlean (1991), «Cognition, enactment and learning in the entrepreneurial process », Frontiers of Entrepreneurship Research, Babson College, p. 242-253.

HAY, M., P. Verdin et P. Williamson (1993), « Successful new ventures : lessons for entrepreneurs and investors », Long Range Planning, vol. 26, $\mathrm{n}^{\circ} 5$, p. 31-41.

HERNANDEZ, E. (1994a), «Le créateur d'entreprise et la démarche stratégique », Direction et Gestion, $\mathrm{n}^{\circ} 146$, p. 19-25 ; n ${ }^{\text {os }} 147-148$, p. 117-128.

HERNANDEZ, E. (1994b), «Modèles d'entrepreneurship : fondamentalisme, contingence et processus », Cahiers du CEDIMES, Université de Paris II, nº 19.

HERRON, L. et R. ROBINSON (1993), «A structural model of the effects of entrepreneurial characteristics on venture performance », Journal of Business Venturing, vol. 8, $\mathrm{n}^{\circ} 3$, p. 281-294.

HOFER, C. et W. BYGRAVE (1992), «Researching entrepreneurship », Entrepreneurship Theory and Practice, Baylor University, vol. 16, no 3, p. 91-100.

HoRnSBy, J., D. NAFFZIGER, D. KURATKO et R. MONTAGNO (1993), « An interactive model of the corporate entrepreneurship process », Entrepreneurship Theory and Practice, Baylor University, vol. 17, $\mathrm{n}^{\circ}$ 2, p. 29-37.

JOHNSON, B. (1990), « Towards a multidimensional model of entrepreneurship, the case of achievement motivation and the entrepreneur », Entrepreneurship Theory and Practice, Baylor University, vol. 14, no 3, p. 39-54.

Julien, P. et M. Marchesnay (1987), La petite entreprise. Principes d'économie et de gestion, Paris, Vuibert, 288 p.

Julien, P. et M. MARCheSNAY (1996), L'Entrepreneuriat, Paris, Economica, 112 p.

KATZ, J. et W. GARTNER (1988), « Properties of emerging organizations », Academy of Management Review, vol. 13, $\mathrm{n}^{\circ}$ 3, p. 429-441.

KEATS, B. et J. BRACKER (1988), «Toward a theory of small firm performance : a conceptual model », American Journal of Small Business, University of Baltimore, vol. $12, \mathrm{n}^{\circ} 4$, p. 41-58.

Khandwalla, P. (1977), The Design of Organizations, New York, Harcourt, Brace and Jovanovich. 
KIRCHLOFF, B. et B. PHILIPPS (1989), «Are small firms still creating jobs ?», Frontiers of Entrepreneurship Research, Babson College, p. 335-349.

LARSON, A. et J. STARR (1993), «A network model of organization formation», Entrepreneurship Theory and Practice, Baylor University, vol. 17, $\mathrm{n}^{\circ} 2$, p. 5-15.

LEARNED, K. (1992), «What happened before the organization? A model of organization formation », Entrepreneurship Theory and Practice, Baylor University, vol. 17, $\mathrm{n}^{\circ}$ 1, p. 39-48.

LIVIAN, Y. et S. MARION (1991), « De l'évaluation des projets de création d'entreprise au pronostic de succès », Revue Internationale PME, vol. 4, nº 1, p. 107-129.

MARCHESNAY, M. (1992), «Les stratégies de spécialisation », Encyclopédie du Management, p. 107-129, Paris Vuibert.

MCDOUgall, P. et R. RoBINSON (1990), «New venture strategies, an empirical identification of eight archetypes of competitive strategies for entry », Strategic Management Journal, vol. 11, p. 447-467.

MCDougall, P., R. RoBINSON et A. DENISI (1992), « Modeling new venture performance : an analysis of new venture strategy, industry structure and venture origin », Journal of Business Venturing, vol. 7, $\mathrm{n}^{\circ}$ 4, p. 267-289.

MILLER, D. et J. TOULOUSE (1986), « Strategy, structure, CEO personality and performance », American Journal of Small Business, University of Baltimore, vol. 10, $\mathrm{n}^{0}$ 3, p. 47-62.

PenRose, E. (1959), The Theory of the Growth of the Firm, Oxford, Blackwell, c1959, 272 p.

SANDBERG, W. (1992), «Strategic management potential contributions to a theory of entrepreneurship », Entrepreneurship Theory and Practice, Baylor University, vol. 16, $\mathrm{n}^{0} 3$, p. 73-90.

SANDBERG, W. et C. HofER (1982), «A strategic management perspective on the determinants of new venture success », Frontiers of Entrepreneurship Research, Babson College, p. 204-237.

SANDBERG, W. et C. HOFER (1986), « The effects of strategy and industry structure on new venture performance », Frontiers of Entrepreneurship Research, Babson College, p. 244-266.

SANDBERG, W. et C. HOFER (1987), «Improving new venture performance: the role of strategy, industry structure, and the entrepreneur », Journal of Business Venturing, $\mathrm{n}^{\circ} 2$, p. 5-28.

SAPIENZA, H., L. HERRON et J. MENENDEZ (1991), « The founder and the firm : a qualitative analysis of the entrepreneurial process ", Frontiers of Entrepreneurship Research, Babson College, p. 254-270.

SAPORTA, B (1994), «La création d'entreprise : enjeux et perspectives », Revue Française de Gestion, $\mathrm{n}^{\circ} 101, \mathrm{p} .74-86$.

SHAPERO, A. (1975), « The displaced, uncomfortable entrepreneur », Psychology Today, vol. $9, \mathrm{n}^{\circ} 6$, p. 83-88.

SHAVER, K. et L. SCOTT (1991), «Person, process, choice: the psychology of new venture creation », Entrepreneurship Theory and Practice, Baylor University, vol. 16, $\mathrm{n}^{\circ} 2$, p. 23-45. 
SHRADER, R. et M. SIMON (1997), « Corporate versus independant new venture : resource, strategy, and performance differences », Journal of Business Venturing, vol. 12, $\mathrm{n}^{\circ} 1$, p. 47-66.

SCHUMPETER, J. (1934), The Theory of Economic Development, Cambridge, Harvard University Press.

SLEVIN, D. et J. CoviN (1995), « New ventures and total competitiveness : a conceptual model, empirical results, and case study examples », Frontiers of Entrepreneurship Research, Babson College, p. 574-588.

STARR, J. et N. FONDA (1992), «A model of entrepreneurship socialization and organization formation », Entrepreneurship Theory and Practice, Baylor University, vol. 17, $\mathrm{n}^{\circ} 1$, p. 67-76.

Stearn, T., N. Carter, P. Reynolds et M. Williams (1995), « New firm survival : industry, strategy, and location », Journal of Business Venturing, vol. 10, $\mathrm{n}^{\circ} 1$, p. 23-42.

STONER, C. (1987), «Distinctive competence and competitive advantage », Journal of Small Business Management, vol. 25, p. 33-39.

TheVenet, M. et J. VACHetTe (1992), Culture et comportement, Paris, Vuibert, 241 p.

VANDEWERF, P. (1993), «A model of venture creation in new industries », Entrepreneurship Theory and Practice, Baylor University, vol. 17, $\mathrm{n}^{\circ}$ 2, p. 39-47.

Woo, C., CoOper, A., DunkelberG, W. et C. Nicholls-NiXon (1990), «Adaptation by start-up firms », Frontiers of Entrepreneurship Research, Babson College, p. 132-143.

WoOdWARD, J. (1965), Industrial Organization: Theory and Practice, Oxford, Oxford University Press.

ZAHRA, S. (1996), «Technology strategy and financial performance: examining the moderating role of the firm's competitive environment », Journal of Business Venturing, vol. 11, no 3 , p. 189-219. 